\title{
bZIPs and WRKYs: two large transcription factor families executing two different functional strategies
}

\author{
Carles M. Llorca ${ }^{\dagger}$, Maren Potschin ${ }^{\dagger}$ and Ulrike Zentgraf* \\ Department of General Genetics, Center of Plant Molecular Biology, University of Tübingen, Tübingen, Germany
}

\section{Edited by:}

Bernd Mueller-Roeber, University of

Potsdam, Germany

Reviewed by:

Sudip Kundu, University of Calcutta, India

Oren Tzfadia, Weizmann Institute of Science, Israel

\section{*Correspondence:}

Ulrike Zentgraf, Department of

General Genetics, Center of Plant

Molecular Biology, University of

Tübingen, Auf der Morgenstelle 32,

72076 Tübingen, Germany

e-mail: ulrike.zentgraf@uni-

tuebingen.de

${ }^{\dagger}$ Carles M. Llorca and Maren Potschin

have contributed equally to this work.
bZIPs and WRKYs are two important plant transcription factor (TF) families regulating diverse developmental and stress-related processes. Since a partial overlap in these biological processes is obvious, it can be speculated that they fulfill non-redundant functions in a complex regulatory network. Here, we focus on the regulatory mechanisms that are so far described for bZIPs and WRKYs. bZIP factors need to heterodimerize for DNA-binding and regulation of transcription, and based on a bioinformatics approach, bZIPs can build up more than the double of protein interactions than WRKYs. In contrast, an enrichment of the WRKY DNA-binding motifs can be found in WRKY promoters, a phenomenon which is not observed for the bZIP family. Thus, the two TF families follow two different functional strategies in which WRKYs regulate each other's transcription in a transcriptional network whereas bZIP action relies on intensive heterodimerization.

Keywords: bZIPs, WRKYs, DNA-binding, heterodimerization, regulatory mechanisms, G/C box accumulation, W-box accumulation

\section{INTRODUCTION}

Due to their sessile nature, plants cannot move to avoid unfavorable conditions as animals do, thus they are forced to cope with their immediate environment, whatever this is. Since the potential environmental variability covers a continuum range from the optimal growth conditions to the toughest stress, a complementary number of possible physiological responses have evolved in order to respond in the most convenient manner to any possible scenario. This process involves transcription factor (TF) networks modulating the expression of a huge number of responding genes.

Unraveling how these networks operate is a major field in plant research, since the comprehensive understanding of the regulatory circuits will allow us to modify them in a beneficial way in the current context of growing food demand and global climate change. Many efforts are focused on deciphering the structure of specific networks by identifying up- and downstream components,

Abbreviations: ABAR, magnesium-protoporphyrin IX chelatase $\mathrm{H}$ subunit $(\mathrm{CHLH}) /$ putative $\mathrm{ABA}$ receptor; $\mathrm{ABF}, \mathrm{ABA}$ responsive element binding factor; $\mathrm{ABI}$ ABA-insensitive; AREB, abscisic acid responsive elements-binding protein; BBX25, B-box domain protein 25; bZIP, basic region/leucine zipper; CCA1, circadian clock associated 1; CKII, casein kinase II; COP1, constitutive photomorphogenic 1; EEL, enhanced em level; EmBP-2, ABRE-binding factor Embp-2 (Zea mays); FLS2, flagellin-sensitive 2; GBF, G-box binding factor; HDA19, histone deacetylase 19; HIS1, histone H1; HY5, elongated hypocotyl 5; HYH, HY5 homolog; ICS1, isochorismate synthase 1; LSD1, lesions simulating disease resistance 1; MEKK, MAP kinase kinase kinase; MKK, MAP kinase kinase; MPK, MAP kinase; NPR1, nonexpressor of PR genes 1; OREB1, ABRE binding factor OREB1 (Oryza sativa); PR1, pathogenesis related 1; RBCS1a, ribulose bisphosphate carboxylase small chain 1a; SIB, sigma factor-interacting protein; SIPK, salicylic acid-induced protein kinase; SnRK, SNF1-related protein kinase; $S U V H 2$, su(var)3-9 homolog 2; TGA, TGA factor family; TRAB1, ABRE binding factor TRAB1 (Oryza sativa); WRKY, WRKY transcription factor; YAP, yeast AP-1-like transcription factor; ZmBZ-1, bZIP transcription factor 1 (Zea mays). however, the comparative analysis of the general features of the regulation of whole families of TFs is still challenging. Granted that TFs within the same family are evolutionary closely related, they are likely regulated by common mechanisms. The recognition of these strategies, shared by entire families of TFs, can provide useful clues to better characterize the function of members of these families.

In this review, we summarize the major regulatory mechanisms characterized so far for WRKYs and bZIPs, two of the largest TF families in plants. Although they have a comparable size, 75 bZIPs and 76 WRKYs can be found in the TAIR database, and they regulate critical physiological processes, such as plant defense, stress responses, or development including senescence; they appear to follow different regulatory strategies. Whereas WRKYs are strongly regulated at the transcriptional level by each other, bZIPs are regulated predominantly at the post-translational level via the formation of heterodimers. This distinction can be inferred from a bioinformatics approach whereby all the Arabidopsis bZIPs and WRKYs IDs gathered from the TAIR were used as an input for the Arabidopsis Interaction Viewer in the BAR webpage (http://bar.utoronto.ca/welcome.htm). The 76 WRKYs resulted in 170 interactions, while the 75 bZIPs yielded in 389 , more than the double than WRKYs. In addition, the WRKY binding motifs (W-boxes) are found to be enriched in the WRKY gene promoters compared to the average occurrence over all Arabidopsis genes. In comparison, C- and G-boxes, the preferred bZIP binding motifs in plants, are not enriched in the bZIP promoters.

\section{THE bZIP TFS AND THEIR REGULATION}

This family of dimeric TFs is present in all eukaryotes, from Saccharomyces cerevisiae (17 bZIP genes) to human (56 bZIP genes). 
bZIPs have been described in Arabidopsis (75), rice (89), sorghum (92), soybean (131), and recently in maize (125; Wei et al., 2012). In plants, they are involved in important processes such as pathogen defense (Alves et al., 2013), abiotic stress signaling (Fujita et al., 2005), hormone signaling (Choi et al., 2000), energy metabolism (Baena-González et al., 2007), as well as development, including flowering (Abe et al., 2005), senescence (Smykowski et al., 2010), and seedling maturation (Alonso et al., 2009).

The name of the family is derived from the basic region/leucine zipper (bZIP) domain present in all its members. This domain consists of an uninterrupted $\alpha$-helix comprising a basic region (BR) which is necessary and sufficient to bind the DNA, followed by a C-terminal leucine zipper (LZ) motif responsible for the dimerization (Schumacher et al., 2000; Miller et al., 2003). The bZIP family was subdivided according to sequence similarities and functional features resulting in 10 groups named A to I, plus S in Arabidopsis (Jakoby et al., 2002; Nijhawan et al., 2007; Wei et al., 2012). While many bZIPs can form homodimers, bZIP members classified in different groups can be combined through heterodimerization to form specific bZIP pairs with distinct functionalities.

\section{THE bZIP STRUCTURE DETERMINES THE DIMERIZATION SPECIFICITY}

By means of dimerization, a limited number of monomers can generate a wide pool of different dimers with singular properties, thereby expanding the repertoire of regulatory responses (Amoutzias et al., 2006, 2008). However, protein interaction has to be selective in order to grant the appropriate response to each situation. In agreement to that, Newman and Keating (2003) showed that, in human and yeast, only $15 \%$ of all possible interactions actually take place between bZIP proteins. This specificity relies on the constitution of the LZ, which is composed of structural repetitions of the so called heptads. In each heptad, seven amino acids are arranged around two $\alpha$-helix turns, in which two definite positions are occupied by leucines or other hydrophobic amino acids. These residues expose their side chains to the same side of the helix, thus resulting in an amphipathic structure. Based on this conformation, hydrophobic forces created between the non-polar sides of two LZs drive their dimerization (Vinson et al., 2002). However, the remaining composition of the heptad is decisive in determining if the interaction will actually take place.

Understanding the forces governing the bZIP dimerization has been a field of intensive research in recent years. To this end, the amino acid positions within a heptad are designated by a specific nomenclature with a letter ranging from $\boldsymbol{a}$ to $\boldsymbol{g}$ (Deppmann et al., 2006). According to that, positions $\boldsymbol{d}$ and $\boldsymbol{a}$ carry the hydrophobic residues and define the hydrophobic face; whereas positions $\boldsymbol{b}, \boldsymbol{c}$, and $\boldsymbol{f}$ are located on the opposite side, the hydrophilic one (Figure 1). Based on this codification and the already described interactions, rules governing the interaction have been formulated (Vinson et al., 2002) and even methods for dimer predictions have been created (Fong et al., 2004). Accordingly, the amino acids in positions $\boldsymbol{a}, \boldsymbol{d}, \boldsymbol{e}$, and $\boldsymbol{g}$ are the ones with a greater impact on determination of the specificity of the interaction (Deppmann et al., 2004). First, the primary hydrophobic forces are established between $\boldsymbol{a}$ and $\boldsymbol{d}$ positions of a heptad and their counterparts in

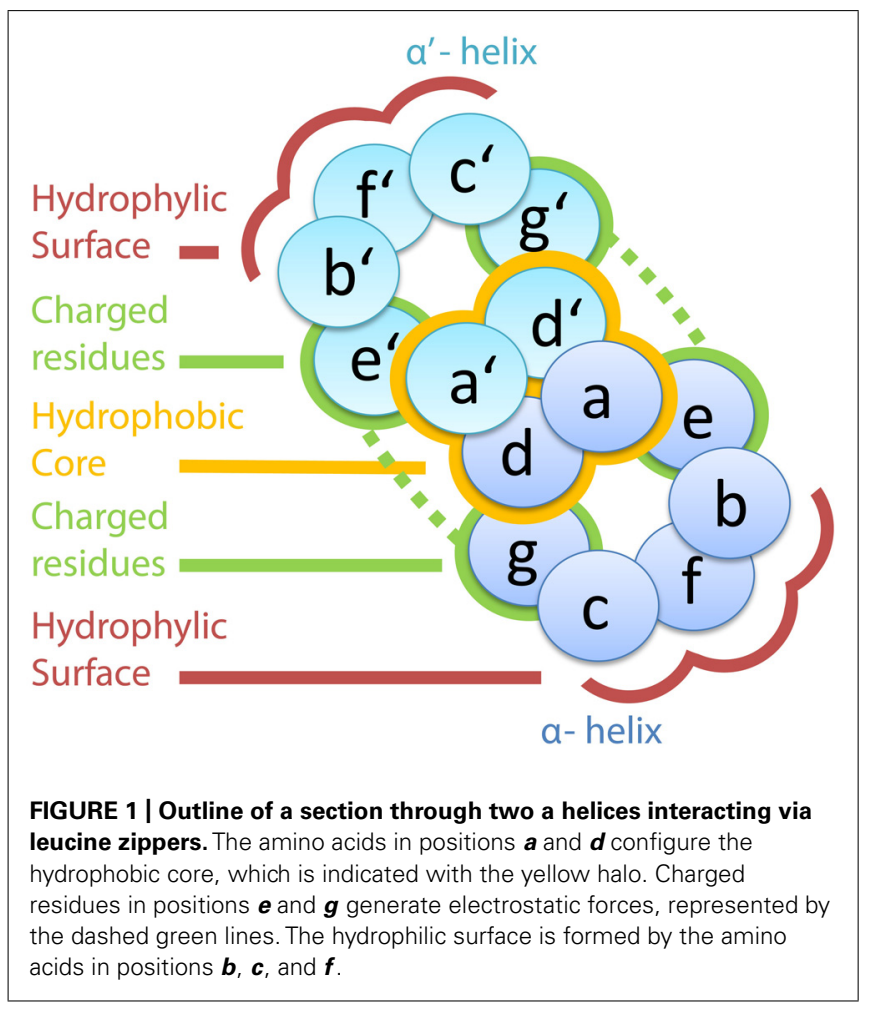

the other LZ disposed in parallel (Vinson et al., 2002), in which the presence of leucines in the $\boldsymbol{d}$ positions is the most stabilizing factor for the dimerization (Moitra et al., 1997). Next, $\boldsymbol{a}-\boldsymbol{a}^{\prime}$ interactions contribute in determining the homodimerizing partners: asparagine residues in this position tend to interact rather with another asparagine, thus favoring the homodimer formation. Conversely, if this position is occupied by a lysine or serine, the heterodimer is favored as these two amino acids prefer residues other than themselves (Acharya et al., 2002). In addition, positions $\boldsymbol{e}$ and $\boldsymbol{g}$ are stabilizing the helix. These two positions act crosswise, so that $\boldsymbol{e}$ positions of one helix interact with $\boldsymbol{g}$ positions on the other one, and usually carry charged or polar amino acids. As a consequence, depending on the charge of these residues, attractive or repulsive forces are formed between the two LZs (Krylov et al., 1994). Overall, the amino acid composition of the LZ determines the energy of the interaction, making each dimer combination more or less likely to happen (Vinson et al., 2006).

Under the above mentioned rules, Arabidopsis bZIPs are predicted to form, almost exclusively, homodimers or quasihomodimers (dimers between two paralogs; Deppmann et al., 2006). Dimerization between bZIPs belonging to the G group (Shen et al., 2008), H group (Holm et al., 2002) or A group (Bensmihen et al., 2002) are in agreement with these predictions. In addition, bZIP are also able to heterodimerize specifically, as the following examples illustrate. The E group members bZIP34 and bZIP61 are unable to homodimerize due to the presence of a proline residue in their LZ, nevertheless they do form heterodimers with the bZIP51 (I group) or the bZIP43 (S group; Shen et al., 2007); G-box binding factor 4 (GBF4), belonging to the A group, interacts with members of the G group (Menkens and Cashmore, 1994); members of the 
$\mathrm{H}$ group can heterodimerize with the G group bZIP GBF1 (Babu Rajendra Prasad et al., 2012 ); or even a whole heterodimerization network involving bZIPs from $\mathrm{C}$ and $\mathrm{S}$ groups has been described (Ehlert et al., 2006).

The preference of bZIPs to interact with more related partners reflects the selectivity of the dimerization. Because they perform similar, even overlapping, functions and can bind to the same ciselements (Jakoby et al., 2002); they can interact laxly, for their ultimate function is not altered to a great extent. In contrast, heterodimerization between bZIPs which are more evolutionary distant is more restricted, as it brings together monomers with more disparate properties. Therefore, the specificity of the partner selection is of central importance because the composition of the dimer will define decisive functionalities such as transactivation potential or DNA-binding activity.

\section{THE bZIP DIMER COMPOSITION DETERMINES THE DNA BINDING}

The DNA recognition by bZIPs takes place with the two continuous $\alpha$-helices wrapped around their LZ regions and pulled apart slightly on their N-terminus, forming a Y-shaped structure which embraces the DNA duplex (Figure 2). In this complex, each BR contacts the DNA along the major groove on opposite sides of the double helix, so that each monomer binds one-half of the DNA target sequence (Glover and Harrison, 1995). As a consequence of the dimeric arrangement of the bZIPs, the binding properties of each dimer are determined by its singular monomer composition.

The target sequences preferentially bound by bZIPs are palindromic or pseudo-palindromic hexamers with an ACGT core (Foster et al., 1994). The positions within a hexamer are designated with a number as established by Oliphant et al. (1989). Under this

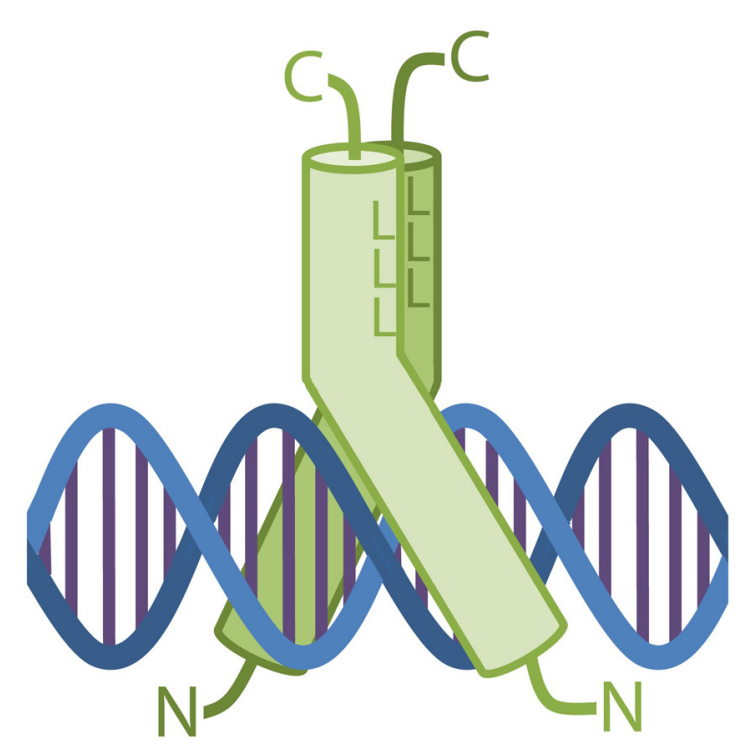

FIGURE 2 | Schematic drawing of a bZIP dimer bound to the DNA. The two proteins form a Y-shape structure which embraces a perpendicularly disposed DNA molecule. The major groove is contacted by both bZIPs via their DNA-binding domains. $L$ represents the leucines forming the interface in the bZIP dimer. code, the bases are given a number radiating from the central positions, i.e., CG, which are both referred as 0 . So, the $5^{\prime}$ half of the sequence are negative values, while the $3^{\prime}$ half are positive. Based on the nucleotide position +2 , different kinds of ACGTcontaining elements are classified as A-box, C-box, G-box, or T-box; among which $\mathrm{C}$ and/or G-boxes are preferentially bound by plant bZIPs (Izawa et al., 1993). Furthermore, the protein binding affinity is determined to a great extent by the nucleotides flanking the hexamer (Williams et al., 1992). The specificity of the DNA recognition arises, thus, as a result of variations in the cis-element sequence combined with the existence of unique BRs mixtures able to discriminate them.

Where exactly the specificity of the interaction relies on has been revealed from solved structures of bZIPs bound to DNA. The target sequence is contacted by only five residues in each BR all along $12 \mathrm{bp}$ in the major groove and these contacts are extended by water molecules (Fujii et al., 2000). These key positions form part of an invariant sequence of nine amino acids $(\mathrm{N}-\mathrm{X} 7-\mathrm{R} / \mathrm{K})$ which feature the BR. Granted that the BR is the most conserved region in bZIPs, the binding preferences of each monomer are determined by only subtle differences in its sequence. For instance, in mouse, bZIPs belonging to the CCAAT/enhancer-binding proteins (C/EBP) family, carry a valine residue in the position 5 of the signature sequence which discriminates against purines at position -3 of the DNA binding site (Miller et al., 2003). Therefore, unspecific interactions with similar DNA sequences are prevented by this single residue. In another case, in the AP-1-like TF (YAP) and CAMP response element binding protein-2 (CREB2) subfamilies of bZIPs in Schizosaccharomyces pombe, the presence of a hydrophobic residue in position 8 of the invariant sequence favors the contact with their AT-rich binding site targets (Fujii et al., 2000).

Besides the identity of amino acids directly contacting the DNA, the specificity of the DNA recognition is further modified by functional variability of the amino acids in the BR. This means that they can adopt different conformations depending on the accompanying residues, which creates a different set of contacts with the DNA bases (Miller et al., 2003). Beyond the BR, it is also known that the hinge region, the junction between the $\mathrm{LZ}$ and the BR, participates in determining the DNA-binding specificity (Niu et al., 1999). Likewise, the presence of ions between the dimer and the DNA (Schumacher et al., 2000), the redox status (Shaikhali et al., 2012), or even the DNA flexibility (Konig and Richmond, 1993) also affect the DNA-binding. On top of that, the BR is intrinsically unstructured in absence of DNA and the folding is only induced upon association with the double helix (Seldeen et al., 2008). Such lack of definite conformation allows the interaction with multiple cis-elements and facilitates post-translational modifications by better exposing the lateral chains, enhancing the regulatory possibilities (Dyson and Wright, 2005).

Granted that the specificity of the DNA recognition arises from the contribution of each BR individually, heterodimerization determines the manner in which the bZIP pairs recognize their target sequences. Through specific heterodimer formation, for example, the binding activity of bZIP53 to the albumin 2S2 promoter is significantly enhanced when combined with bZIP25 or bZIP10 (Alonso et al., 2009). Conversely, other bZIPs lose their 
DNA-binding when associated to particular partners, as bZIP1, whose DNA-binding activity is prevented in combination with bZIP63 or bZIP10 (Kang et al., 2010).

\section{THE DIMER COMPOSITION DETERMINES THE TRANSACTIVATION PROPERTIES}

In addition to their role in the DNA recognition, each monomer contributes individually to the transactivation capacity (Miotto and Struhl, 2006). While some bZIPs have special domains acting as transactivators or as repressors, e.g., the proline rich domain in the G group (Shen et al., 2008), others require the presence of additional elements, such as coactivators (Rochon et al., 2006) or histone deacetylases (Kuo et al., 2000). Besides, the transactivation activity of the same bZIP can be further modified through the interaction with other proteins, e.g., the transactivation capacity of ELONGATED HYPOCOTYL 5 (HY5) is enhanced by the clock protein CCA1 (Andronis et al., 2008), but is inhibited when interacting with BBX25 (Gangappa et al., 2013). As a result of the peculiar transactivation properties of each bZIP, the composition of the dimer determines the outcome of target gene expression.

More important, if different dimers can affect the expression in a peculiar manner, they can compete for the same cis-element with other bZIP pairs, constituting an efficient mechanism to adjust the expression of a given gene. Such a system has been described controlling the expression of late-embryogenesis abundant genes in Arabidopsis by the A group bZIPs ABA-insensitive 5 (ABI5) and EEL. These two bZIPs compete for the same binding site, conferring antagonistic transactivation functions: ABI5 homodimers activate the gene expression, whereas EEL homodimer and ABI5-EEL heterodimer repress it (Bensmihen et al., 2002). Furthermore, gradation of the expression can be achieved through the formation of different heterodimers. So is the expression of RBCS1a modulated by HY5, HY5 homolog (HYH), and GBF1 in which GBF1 acts a repressor, HY5 and HYH act as inducers. However, the different heterodimers that can be formed show intermediate effects depending on the pair of monomers combined (Singh et al., 2012). In other cases, functional cooperation between monomers is established instead of competition. Indeed, heterodimerization appears to be a requirement for the induction of genes under control of bZIPs belonging to the C/S1 network. In other words, while these bZIPs are not able to activate the gene expression by themselves alone, certain heterodimers result in a strong activation of specific target genes (Weltmeier et al., 2006).

\section{MONOMER AVAILABILITY IS A HOT SPOT IN bZIP REGULATION}

Having established how relevant the identity of the monomers in each dimer is, the availability of monomers arises as a key point of regulation restricting the number of interactions that can take place. The expression of bZIP genes is, indeed, adjusted to control their abundance, showing tissue specificity (Fujita et al., 2005; Iwata et al., 2008; Weltmeier et al., 2008; Alonso et al., 2009), as well as developmentally regulated expression, including embryogenesis (Bensmihen et al., 2002; Weltmeier et al., 2008), flowering (Abe et al., 2005), or senescence (Breeze et al., 2011). Furthermore, changes in expression of bZIPs have been reported upon exposure to certain stresses. For example, $\mathrm{Zn}$ deficiency increases the transcription of bZIP23 and bZIP19 (Assunção et al., 2010), bZIP53 and bZIP10 are induced after an osmotic stress period (Weltmeier et al., 2006). The ABA-responsive element binding protein (AREB) subfamily of bZIPs is up-regulated by drought and salt in Arabidopsis (Uno et al., 2000) as well as in tomato (Hsieh et al., 2010; Orellana et al., 2010). Besides, a remarkable amount of studies relate changes in the bZIP expression to the energy status. These include the repression of bZIP1 and bZIP63 by sugars (Kang et al., 2010; Matiolli et al., 2011) and bZIP11 by darkness (Rook et al., 1998), or the induction of the expression of several bZIPs by the activation of the energy deficiency-related kinase SNF1-related protein kinase 1 (SnRK1; Baena-González et al., 2007). Beyond the transcriptional level, bZIPs are regulated by alternative splicing (Zou et al., 2007) and by controlling the translation initiation, e.g., the repression of translation by sucrose in bZIPs belonging to the subgroup S1 in Arabidopsis (bZIP1, bZIP2, bZIP11, bZIP44, bZIP53; Wiese et al., 2004; Weltmeier et al., 2008).

After the protein synthesis, specific control of the protein turnover has been found regulating the abundance of some bZIPs such as GBF1 (Mallappa et al., 2008), ABF1 and ABF3 (Chen et al., 2013), or TGAs (Pontier et al., 2002). In addition, the amount of functionally active monomers in the nucleus is regulated by subcellular partitioning. In order to be targeted to the nucleus, bZIPs carry a nuclear localization signal (NLS) which is located within the $\mathrm{BR}$, overlapping with the invariant DNA binding sequence and consisting of two clusters of lysines/arginines (Miller, 2009). Nevertheless, few bZIPs have been found outside of the nucleus being retained by different means. For instance, bZIP10 is retained in the cytoplasm by the zinc-finger protein lesions simulating disease resistance 1 (LSD1). This protein interferes with the NLS-mediated nuclear import of bZIP10 (Kaminaka et al., 2006). In other cases, bZIPs are actively shuttled out of the nucleus due to the presence of a nuclear export signal (NES; Tsugama et al., 2012) and they stay in the nucleus only when the NES gets masked (Li et al., 2005). Finally, extra-nuclear retention can be achieved by attachment to membranes. The so called membrane associated bZIPs are anchored via an N-terminal trans-membrane domain and are transferred to the nucleus after proteolytic cleavage. In Arabidopsis, bZIP17 (Liu et al., 2008), bZIP28 (Liu et al., 2007a), and bZIP60 (Iwata et al., 2008) have been found to be membrane associated so far.

\section{bZIP ACTIVITY IS BROADLY REGULATED BY PHOSPHORYLATION}

The activity of the available bZIP monomers can be further regulated by phosphorylation. This kind of post-translational modification can modify all the above-mentioned mechanisms controlling the TF function. First, dimerization specificity can be altered through phosphorylation of the LZ (Lee et al., 2010). Next, the DNA-binding of the bZIPs to their target sequences can be prevented by the addition of a phosphate group into the $\mathrm{BR}$, which contributes with a negative charge creating repulsive forces with the DNA molecule (Deppmann et al., 2003; Kirchler et al., 2010). Besides, phosphorylation within other regions of the protein can trigger conformational changes required for the activation of the protein (Lee et al., 2010). In addition to the 
direct effect on the bZIP activity, phosphorylation can adjust the monomer abundance by altering the protein turnover. For example, phosphorylation of $\mathrm{ABF} 3$ creates a binding site for a 14-3-3 protein which protects $\mathrm{ABF} 3$ from rapid turnover (Sirichandra et al., 2010). Likewise, phosphorylation of HY5 prevents its degradation by impeding the interaction of this bZIP with the E3-ubiquitin-protein ligase COP1 (Hardtke et al., 2000). Finally, phosphorylation can control the bZIP subcellular localization, targeting a bZIP either for nuclear import (Djamei et al., 2007) or for cytoplasmic retention (Ishida et al., 2008).

Above all, the manner in which the activity of a bZIP is regulated is specific meaning that the same kinase enhances the activity of some bZIPs, but diminishes the action of others. Such a situation has been described for instance for EmBP-2 and ZmBZ-1 phosphorylated by CKII (Nieva et al., 2005). The specific effect of the phosphorylation for each bZIP allows the customized regulation of multiple genes by the action of few upstream kinases. This is an optimal feature for the control of responsive pathways and, indeed, bZIPs are frequently found to be involved in such networks like, e.g., the deciphered ABA-responsive pathway in rice, which involves the action of a SnRK, namely SnRK2, activating the transcription of the $\mathrm{ABA}$ responsive genes through the phosphorylation of the bZIP proteins OREB1 and TRAB1 (Kagaya et al., 2002; Kobayashi et al., 2005; Chae et al., 2007). Similarly, bZIPs belonging to the $\mathrm{S}$ and $\mathrm{C}$ groups coordinate the activation of the metabolic response to low energy stress in combination with SnRK1 (BaenaGonzález et al., 2007; Hummel et al., 2009; Dietrich etal., 2011; Cho et al., 2012).

\section{THE WRKY TFs AND THEIR REGULATION}

The WRKY TF family is found in the plant kingdom and belongs also to the 10 largest families of TFs in higher plants. Like bZIPs, the WRKY family is divided into different subgroups, but in contrast to the ten bZIP groups, the WRKY family is only divided into three groups. WRKY factors are also found in the unicellular eukaryote Giardia lamblia and the slime mold Dictyostelium discoideum (Ulker and Somssich, 2004; Zhang and Wang, 2005), but there is no hint that WRKY TFs exist in animals. However, former analyses have shown that WRKY TFs belong to a WRKYGCM1 (glial cell missing 1) superfamily which is a widespread eukaryote-specific group of TFs (Babu et al., 2006).

Almost two decades have already passed since their discovery (Ishiguro and Nakamura, 1994; Rushton et al., 1995, 1996) and by now a lot of different functions have been attributed to the WRKY TFs. They participate in the regulation of many plant processes including the responses to pathogen infestation (Pandey and Somssich, 2009; Birkenbihl et al., 2012; Hu et al., 2012; Chujo et al., 2013), abiotic stresses (Jiang and Deyholos, 2009; Rushton et al., 2010; Scarpeci etal., 2013; Wang et al., 2013), trichome development (Johnson et al., 2002), and senescence (Zentgraf et al., 2010; Zhou et al., 2011; Besseau et al., 2012). Northern blot analysis revealed that in Arabidopsis around 70\% of the WRKY genes were differentially expressed in plants after infestation with an avirulent strain of the bacterial pathogen Pseudomonas syringae or treatment with salicylic acid (Dong et al., 2003) emphasizing their importance in pathogen response. A more recently described physiological activity of WRKY factors is their participation in the biosynthesis of alkaloids (Suttipanta et al., 2011; Yamada and Sato, 2013; Yang et al., 2013).

\section{WRKY STRUCTURAL FEATURES}

The WRKY factors are named after their characteristic DNAbinding domain (DBD) of approximately 60 amino acids. This domain contains a highly conserved WRKYGQK motif at the Nterminus and a zinc-finger structure at the C-terminus called the WRKY domain. There are two possibilities how the zinc-finger structure of this domain can be formed, either $\mathrm{Cx}_{4-5} \mathrm{Cx}_{22-23} \mathrm{HxH}$ $(\mathrm{C} 2 \mathrm{H} 2)$ or $\mathrm{Cx}_{7} \mathrm{Cx}_{23} \mathrm{HxC}(\mathrm{C} 2 \mathrm{HC})$, in which the cysteine and histidine residues bind one zinc atom and generate a finger like structure. Both, the WRKYGQK motif and the zinc-finger structure are necessary for the DNA-binding activity of WRKY TFs. Mutations in the invariable WRKYGQK motif significantly reduced the DNA-binding activity and substitutions of the conserved $\mathrm{C}$ and $\mathrm{H}$ residues of the zinc-finger even abolished the DNA-binding (Maeo et al., 2001).

All WRKY proteins contain one or two of these DNA-binding WRKY domains and are categorized into three subgroups dependent on their number of WRKY domains and the zinc-finger structure. Group I WRKY proteins are marked by two WRKY domains with a $\mathrm{C} 2 \mathrm{H} 2$ zinc-finger structure. Group II and III WRKY proteins consist of only one WRKY domain with a $\mathrm{C} 2 \mathrm{H} 2$ and a $\mathrm{C} 2 \mathrm{HC}$ zinc-finger structure, respectively. The group II WRKY proteins were originally further divided into IIa, IIb, IIc, IId, and IIe based on their primary amino acid sequence, but later, phylogenetic analyses have shown, that the subgroups IIa and IIb are combined to IIa + b, and IId and IIe to IId + e (Eulgem et al., 2000; Zhang and Wang, 2005; Rushton et al., 2010).

Recently, it was shown for Solanum lycopersicum that even sequence variants for the highly conserved WRKYGQK motif exist. WRKYGKK is the most common variant, but WRKYGMK, WSKYGQK, WQKYGQK, and WIKYGEN have also been described. Furthermore, it was found that also novel zincfinger variants exist, namely $\mathrm{Cx}_{29} \mathrm{HxH}$ and $\mathrm{Cx}_{7} \mathrm{Cx}_{24} \mathrm{HxC}$ (Huang et al., 2012). Moreover, Mangelsen et al. (2008) could also detect variants of the WRKYGQK motif (WRKYGKK, WQKYGQK, WRKYGEK, and WSKYGQM) in Hordeum vulgare.

The WRKY domain binds to a so called W-box (TTGACC/T) in the promoters of target genes. This sequence is the minimal core element necessary for binding of a WRKY protein to DNA (Rushton et al., 1996; Ciolkowski et al., 2008). W-boxes can be found in the promoters systemic acquired resistance related (SAR) genes, including isochorismate synthase 1, non-expressor of PR genes 1 , and pathogenesis related 1 (Fu and Dong, 2013); or ABA signalingrelated genes such as $A B I 4, A B I 5$, and $A B A$ responsive element binding factor 4 (Rushton et al., 2012). Often there are several Wboxes in one promoter, and even motif clusters can be found. Remarkably, W-boxes are also found in the promoter of WRKY genes, suggesting a potentially strong transcriptional networking between WRKY proteins.

The elucidation of the solution structure of WRKY proteins in contact with the DNA will help to understand the mechanism of DNA-binding. In 2005, the solution structure of the C-terminal WRKY domain of Arabidopsis WRKY4 (a group I WRKY protein) was discovered by NMR (Yamasaki et al., 2005). Yamasaki 


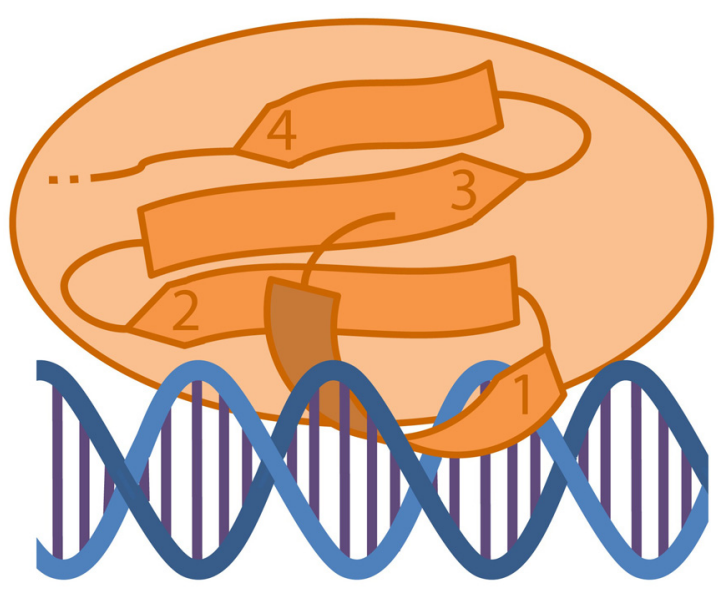

FIGURE 3 | Diagram of a WRKY C-terminal domain interacting with the DNA. The C-terminal WRKY domain consists of a four-stranded antiparallel $\beta$-sheet (1-4). The DNA recognition takes place along the major grove by the $\beta 1$-strand containing the WRKYQK motif.

et al. (2012) could dissolve the structure of the same domain in complex with a W-box. The C-terminal WRKY domain consists of a four-stranded antiparallel $\beta$-sheet, in which the $\beta 1$-strand, that comprises the WRKYGQK motif, contacts the major DNA groove (Figure 3). Residues of the WRKYGQK motif recognize the DNA mainly through apolar contacts with methyl groups of the $\mathrm{T}$ bases of the W-box. The DNA in this model is B-formed. Another model for the protein-DNA structure formation was proposed in 2007 by Duan et al. (2007). They investigated the crystal structure of the WRKY domain of Arabidopsis WRKY1 (also a group I WRKY protein), but the domain attribution used for WRKY1-C was longer. They found that this WRKY domain consists of a five-stranded antiparallel $\beta$-sheet with $\beta 2$ and $\beta 3$ forming the DNA-binding sites. The zinc-binding site was found between $\beta 4$ and $\beta 5$. By using a similar domain attribution like Yamasaki et al. (2012) the structure between WRKY4-C and WRKY1-C was comparable.

\section{THE W-bOx, SURROUNDING SEQUENCES AND THE WRKY DOMAIN DETERMINE THE DNA-BINDING SPECIFICITY}

WRKY TFs bind W-boxes in the promoters of target genes to regulate their expression. But almost all WRKY factors bind Wboxes raising the question, how specificity is achieved between certain promoters and different WRKY TFs.

Binding studies revealed that only the presence of $\mathrm{W}$-boxes is not sufficient for a DNA-protein interaction. By using gel shift experiments, Miao et al. (2004) could show a specific DNAbinding activity of WRKY53 to promoter fragments of its target gene senescence-induced receptor-like kinase in which a complex was only formed with a fragment containing four W-boxes, whereas no DNA-protein interaction was found when the fragment consisting of only three $\mathrm{W}$-boxes. However, reporter gene assays showed, that these three boxes are necessary for the induction of a reporter gene by WRKY6 (Robatzek and Somssich, 2002). This indicates that the presence of $\mathrm{W}$-boxes is not sufficient for specific binding and that most likely the surrounding sequences and the overall structures are important. A more detailed study for five different WRKY TFs toward their DNA-binding selectivity depending on neighboring sequences was performed by Ciolkowski etal. (2008). They found differences in the binding site preferences of WRKY6, WRKY11, WRKY26, WRKY38, and WRKY43 by gel shift experiments. WRKY6 (group IIb) and WRKY11 (group IId) show high binding affinity to sequences with a $\mathrm{G}$ residue directly adjacent $5^{\prime}$ to the W-boxes, whereas WRKY26 (group I), WRKY38 (group III), and WRKY43 (group IIc) bind more efficiently with a $\mathrm{C}, \mathrm{A}$, or $\mathrm{T}$ in the direct $5^{\prime}$ neighborhood. Interestingly, the binding of these three WRKYs was enhanced by exchanging the first $\mathrm{T}$ base in $5^{\prime}$ direction. Ciolkowski et al. (2008) concluded again that for a specific transcriptional regulation the adjacent sequences to $\mathrm{W}$-boxes are important. Besides, there are some reports of WRKY proteins binding to non-Wbox sequences. In a reporter gene assay, WRKY6 can regulate the reporter gene expression under the control of the WRKY42 promoter lacking perfect W-boxes (Robatzek and Somssich, 2002). WRKY53 can also directly interact with a W-box lacking fragment of the same promoter (Miao et al., 2004) and with clustered imperfect W-boxes only consisting of the TGAC core elements of a W-box (Potschin et al., 2013) indicating more diversity in sequence affinities of WRKY TFs. Moreover, binding to a PRE4 element (TACTGCGCTTAGT) and to a W-box containing element was shown for OsWRKY13 of rice (Cai et al., 2008).

A DNA-protein interaction enzyme-linked immunosorbent assay (DPI-ELISA) screen was developed by Brand et al. (2013) to elucidate WRKY DNA-binding specificities in a more general view. They used only the WRKY DBDs for the DPI-ELISAs with the aim to unravel the DNA-sequence specificity for each WRKY DBD. The DBDs of AtWRKY50, AtWRKY11, and AtWRKY33 (Cterminal DBD and N-terminal DBD) were tested and, in fact, they found sequences that seem to be DBD-specific. Remarkably, they could show that both DBDs of group I WRKYs are functional and can bind to DNA, even though the binding of the N-terminal DBD was weaker than that of the C-terminal DBD. Although homology modeling revealed a potential binding ability for both domains, the N-terminal domain always showed weaker or even no binding (Eulgem et al., 1999; Maeo et al., 2001; Duan et al., 2007). However, the actual function of the N-terminal WRKY domain is still unclear.

As mentioned above, there are sequence variants for the highly conserved WRKYGQK motif of the WRKY domain. The Arabidopsis WRKY50 factor has the slightly different amino acid sequence WRKYGKK in the WRKY domain (Eulgem et al., 2000; Brand etal., 2013). Brand etal. (2013) chose the WRKY domain of this WRKY TF and the Arabidopsis WRKY11 DBD with a conserved WRKYGQK motif to investigate, if there is a difference in the DNA recognition caused by this single amino acid exchange (lysine and glutamine) in the DNA-binding site. The amino acid glutamine prefers to bind nucleobases due to its partial negative charge, whereas lysine prefers to bind the phosphate backbone due to its partial positive charge. In fact, these WRKY domains showed preferences for distinct DNA target sequences, depending on this amino acid exchange in the conserved WRKYGQK motif. They mutated the conserved motif of WRKY50 to WRKYGQK ${ }^{(\mathrm{KQ})}$ and this of WRKY11 to WRKYGKK ${ }^{(\mathrm{QK})}$ and tested these mutated 
WRKY domain proteins in DPI-ELISAs. WRKY50 ${ }^{\text {mut }}$ showed a similar DNA-binding affinity like WRKY $11^{\text {wt }}$ and WRKY $11^{\text {mut }}$ like WRKY50 ${ }^{\mathrm{wt}}$, suggesting that these amino acids in the WRKY domain are important for specific DNA recognition.

\section{W-bOXes IN WRKY GENE PROMOTERS ENABLE TRANSCRIPTIONAL NETWORKING}

An interesting point that has emerged in promoter analysis of WRKY TFs is the enrichment of W-boxes in their own promoters as indicated by Dong et al. (2003). They analyzed the $1.5 \mathrm{~kb}$ promoter sequence upstream of 72 WRKY genes in Arabidopsis, finding that $83 \%$ of the WRKY genes contain at least two perfect $\mathrm{W}$-boxes (TTGACC/T) and 58\% contain even four or more TTGAC core elements suggesting a regulatory network between the WRKY factors.

Further detailed studies of several WRKY promoters also confirmed the presence of multiple W-boxes. For example, two W-boxes were found in the promoter of AtWRKY6, four and five W-boxes in the promoters of the two homologous genes of Coffea arabica, five W-boxes in the promoter of AtWRKY18, and three perfect W-boxes plus an additional TGAC cluster in the promoter of AtWRKY53 (Robatzek and Somssich, 2001; Petitot et al., 2013; Potschin et al., 2013). Some WRKYs even carry 11 or 12 (AtWRKY66, AtWRKY17) TTGAC core elements in an analyzed $1.5 \mathrm{~kb}$ promoter fragment (Dong et al., 2003). In order to compare the bZIP family with the WRKY family in this aspect, we analyzed bZIP promoters for C and G-boxes and WRKY promoters for W-boxes. We could easily verify this enrichment analyzing the 3-kb upstream fragments of 76 WRKY genes, which led to similar results: $72 \%$ of the WRKY genes contain two or more Wboxes. Furthermore, we found that $40 \%$ of the WRKYs have three or more W-boxes in their promoters, which is clearly above the average found for all annotations in the TAIR database (Figure 4). In contrast, no enrichment of C- or G-boxes could be detected in the bZIP promoters compared to the overall distribution of these cis-elements.

In agreement with the $\mathrm{W}$-box enrichment in their own promoters, it has been demonstrated that the WRKY proteins act on the promoters of their own genes and on other WRKY genes in cotransfection assays resulting in activation or repression of a reporter gene (Robatzek and Somssich, 2002; Petitot et al., 2013; Potschin et al., 2013). In addition, a pull-down analysis of WRKY53 with genomic DNA resulted in a list of putative target genes of WRKY53 including eight different WRKY genes (Miao et al., 2004). Furthermore, the analysis of wrky mutant or overexpression plant lines revealed that the expression of other WRKY genes is altered in these lines. Loss of the AtWRKY22 protein increased the expression of AtWRKY70 after dark treatment, whereas overexpression of the AtWRKY22 protein decreased the expression of AtWRKY70 under normal conditions in comparison to wild type plants. When AtWRKY70 is mutated, the expression of AtWRKY22 is decreased compared to wild type plants after dark treatment (Zhou et al., 2011). Moreover, a double-knock out mutant of Atwrky11 wrky17 showed increased transcript levels of AtWRKY70 and AtWRKY54 (Journot-Catalino et al., 2006). Microarray analyses of stressed Atwrky33 mutant plants compared to the wild type revealed lower expression of AtWRKY28, which
A

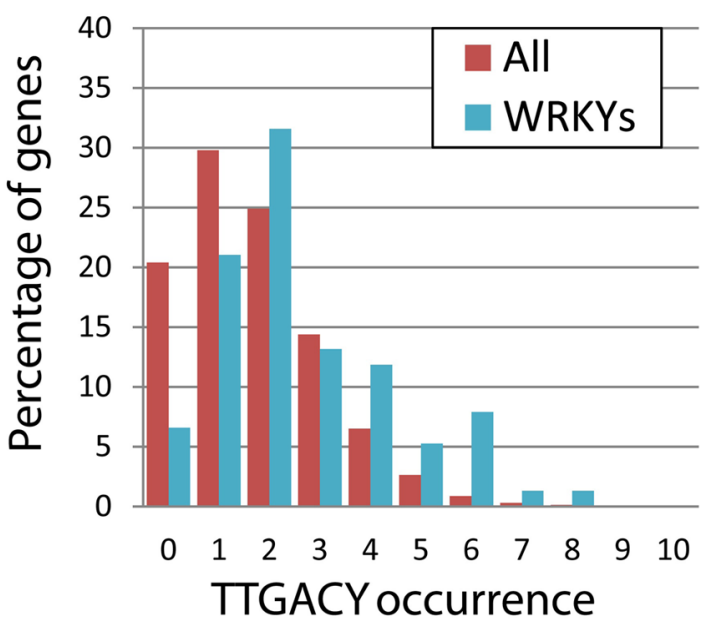

B

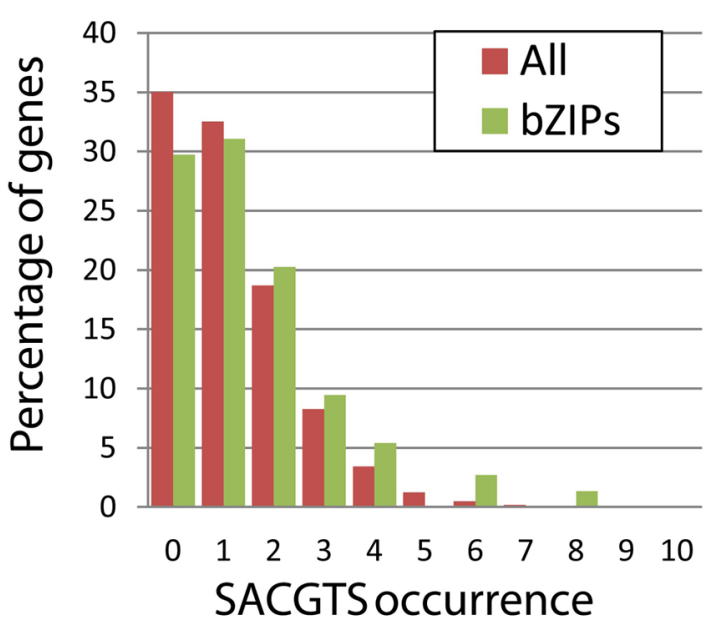

FIGURE 4 | Frequency of cis-element distribution within the 3-kb upstream of coding sequences. The occurrence of the cis-elements was calculated only for the positive strand using the Patmatch tool in the TAIR website. Red bars refer to the whole set of 33,602 annotations of the TAIR10 Genome Release, while green and blue bars indicate the subsets including only bZIPs or only WRKYs genes, respectively. (A) The occurrence of W-boxes was determined using a TTGACY motif, where $Y$ indicates pyrimidine. The enrichment of W-boxes in the WRKY promoters is remarkable, while only few WRKY promoters carry no W-boxes. (B) The occurrence of the G- and C-boxes, the preferred cis-elements bound by plant bZIPS, was determined using a SACGTS motif, where S indicate strong bases ( $\mathrm{C}$ and $\mathrm{G}$ ). In contrast, no increase of bZIP-binding sites in their promoters can be observed.

was also confirmed by qRT-PCR (Jiang and Deyholos, 2009). Alteration on the expression of certain WRKY genes was also shown for Atwrky 18 mutant compared to wild type plants in microarray analyses (Wang et al., 2006). Based on 2000 Arabidopsis microarray experiments, it was found that more than $70 \%$ (45 out of 61) of the WRKY genes are co-regulated with other WRKYs (Berri 
et al., 2009). In addition, ChIP resolution scanning of the parsley PcWRKY1 promoter with an antiserum that detects most of the WRKY factors showed that the W-boxes of this promoter are constitutively occupied by WRKY factors (Turck et al., 2004). Therefore, it seems that the WRKY factors act in a network with mutually regulation of their own expression.

\section{WRKY PROTEINS CAN INTERACT WITH MULTIPLE PARTNERS}

In addition to transcriptional networking, WRKY proteins can also form dimers and are also capable to form heterodimers. Furthermore, many other proteins have been characterized to form protein complexes with WRKY proteins thereby regulating their function. An excellent overview on protein interaction partners of WRKY proteins was recently published by Chi et al. (2013). Here, we focus on the heterodimer formation between WRKY factors and their impact on transcription.

The growing number of discovered interaction partners reveals that there is also a networking between the WRKY factors on the protein level. Moreover, there is some evidence that these WRKY heterodimers act in a different way on transcriptional regulation than homodimers or monomers. Recently, a participation of AtWRKY18 in the senescence process was discovered (Potschin etal., 2013). AtWRKY18 can physically interact with AtWRKY53, an important regulator of early senescence, leading to different transcriptional activation in a reporter gene assay of the heterodimer in comparison to the single proteins. A well-investigated network exists between the three Arabidopsis WRKY factors WRKY18, WRKY40, and WRKY60. It was shown by Xu etal. (2006) that these three WRKYs interact with each other in a yeast two-hybrid assay and form homoand heterodimers. In gel shift assays, WRKY18 and WRKY40 heterodimers bind much stronger to different W-box carrying sequences than the respective homodimers. In contrast, if WRKY40 is mixed with WRKY60 proteins the binding affinity declines. Since WRKY60 alone shows almost no binding activity for the used DNA sequences the effect has to be due to heterodimer formation. An example for the regulation activity of WRKY18/WRKY40 heterodimers is given by Chen et al. (2010). WRKY60 is expressed after ABA treatment and this induction is almost lost in the wrky 18 and wrky 40 mutants, suggesting that WRKY60 is regulated by WRKY18/WRKY40 in the ABA signaling pathway. In addition, they could show activation of the WRKY60 promoter by WRKY18/WRKY40 heterodimers in a reporter gene assay, whereas the homodimers had no effect (Chen et al., 2010). These three WRKY proteins participate in the ABA signaling pathway through direct regulation of ABI4 and ABI5. Interestingly, not only different binding effects to these two genes were observed for the heterodimers, by using fragments of the $\mathrm{ABI} 4$ and $\mathrm{ABI} 5$ promoters in gel shift assays, binding activity of a combination of all three WRKYs together was sometimes completely abolished binding, although all possible heterodimers could bind to the same fragment. This indicates that an interaction between all three WRKY proteins takes place and that this higher order complex has again a distinct functionality (Liu et al., 2012).

An example of different binding activity for heterodimers between WRKYs and non-WRKY proteins is given by Lai et al. (2011). AtWRKY33 can interact with SIGMA FACTORINTERACTING PROTEINS 1 and 2, two VQ motif-containing proteins that stimulate the DNA-binding activity of WRKY33. It was shown in a yeast two-hybrid assay using deletion mutants of WRKY33 that this interaction is mediated by the C-terminal WRKY domain of WRKY33. WRKY33 belongs to group I with the characteristic of two WRKY domains in which in general the C-terminal WRKY domain carries out the DNA-binding. However, the C-terminal WRKY domain is also responsible for mediating protein-protein interactions (Lai et al., 2011), so that these two functions overlap in this domain. Besides, in a yeast two-hybrid screen with Arabidopsis VQ and WRKY proteins, the C-terminal WRKY domain of group I WRKY proteins and the sole WRKY domain of group IIc WRKY proteins seem to be important for protein-protein interactions (Cheng et al., 2012). Group IIa WRKY proteins contain canonical LZ sequences and many other group II and III WRKYs have at least multiple leucine, isoleucine or valine residues at their $\mathrm{N}$-termini, forming a similar structure of a LZ for protein-protein interactions (Chi et al., 2013).

$\mathrm{W}$-boxes in the promoters of target genes are often clustered. Since one WRKY DBD is thought to bind one W-box, such Wbox clusters in the DNA can mediate a complex formation of higher order protein complexes between different WRKY proteins. Depending on the orientation and the number of nucleotides between the W-boxes, the WRKY DNA-binding protein complex is composed of WRKY proteins with specific conformations. However, higher order complex formation does not only refer to clustered W-boxes, but also to separated W-boxes through DNA loop formations (Chi et al., 2013), enhancing again the variety of WRKY TF activity. In contrast to the bZIP factors that need to dimerize for DNA-binding, the mode of DNA-binding seems to be more diverse for the WRKY factors. They appear to bind as monomers, dimers or even as trimers (Xu et al., 2006; Ciolkowski et al., 2008; Liu et al., 2012). But although single WRKY proteins were usually used in gel shift experiments, it is still possible that these WRKYs form homodimers. The isolation of the solution structure of a WRKY protein in complex with DNA revealed that monomer binding occurs, although they did not use the whole protein for structure analysis.

\section{WRKY ACTIVITY IS ALSO MODULATED BY PHOSPHORYLATION BUT THROUGH DIFFERENT KINASES}

As already described for bZIPs, WRKY TFs activity can also be modulated by phosphorylation. In the case of WRKYs, phosphorylation can be mediated through the mitogen-activated protein kinase (MAPK) pathway (Asai et al., 2002). Normally, a MAP kinase kinase kinase (MEKK) phosphorylates and activates a MAP kinase kinase (MKK) that in turn phosphorylates a MAPK responsible for phosphorylation and regulation of different effector proteins. An entire MAPK signaling cascade was characterized for the response of plant cells to the bacterial component flagellin which is sensed by the flagellin receptor FLS2 (flagellin-sensitive 2), a leucine-rich-repeat (LRR) receptor kinase. The MEKK MEKK1 is activated by the FLS2 kinase, MEKK1 activates MKK4/MKK5, two MKKs that activate MPK3/MPK6, two MAPKs that activate the effector proteins WRKY22/WRKY29 resulting in an immune response. Activation of this MAPK cascade 
confers resistance to both bacterial and fungal pathogens (Asai et al., 2002). Phosphorylation-dependent activation in immune responses was also shown for Nicotiana benthamiana WRKY8. NbWRKY8 increases its DNA-binding activity after incubation with salicylic acid-induced protein kinase (SIPK), a MAPK that is also able to phosphorylate NbWRKY8. Additionally, phosphorylation of NbWRKY8 resulted in an enhanced transactivation activity in a reporter gene assay (Ishihama et al., 2011). For its homolog AtWRKY33, a regulation through phosphorylation was also shown. Transcriptomic analysis of wrky33 and wild type plants upon Botrytis cinerea infection discovered a strong transcriptional reprograming mediated by AtWRKY33 in plant pathogen responses (Birkenbihl et al., 2012). AtWRKY33 is a substrate of MPK3/MPK6, two MAPKs important for the induction of camalexin biosynthesis (Ren et al., 2008), the major phytoalexin in Arabidopsis, and is therefore responsible for growth inhibition of certain pathogens (Glawischnig, 2007). Mutation of five potential phosphorylation sites in WRKY33 in the wrky33 mutant background blocks the ability of WRKY33 to restore the induction of camalexin production (Mao et al., 2011). AtWRKY53, a positive regulator of senescence is phosphorylated by MEKK1 although this kinase is upstream in the MAPK signal cascade and appears to take a short cut. The phosphorylation enhances DNA-binding activity of AtWRKY53 in vitro and transcription of a reporter gene in vivo (Miao et al., 2007). Phosphorylation is often mediated by clustered Pro-directed Ser residues (SP-cluster) in the N-terminal region of several group I WRKY proteins. In addition, some group I WRKYs harbor a so called D-domain $\left[(\mathrm{K} / \mathrm{R})_{1-2}-\mathrm{X}_{2-6^{-}}(\mathrm{L} / \mathrm{I})-\mathrm{X}-\right.$ (L/I)] important for the interaction with MAPKs (Ishihama et al., 2011). However, interaction with MAPKs is not restricted to group I WRKY proteins. Popescu et al. (2009) found in protein microarrays a lot of WRKYs from different groups as interaction partners of diverse MAPKs with most of the WRKYs carrying SP-cluster.

\section{WRKY EXPRESSION IS ALSO UNDER EPIGENETIC CONTROL}

In eukaryotic cells, nuclear DNA wraps around histone proteins forming nucleosomes that are finally packaged into chromatin. Whereas euchromatin is the loosely packaged form accessible for the transcription machinery, heterochromatin is tightly packaged and transcriptionally inactive. These two states are not static but can be converted into each other providing an essential mechanism of regulating gene expression. Conversions are predominantly achieved through modifications of the histones by acetylation, methylation, and phosphorylation. Acetylation of a histone results in a more loosely form of the nucleosome and an easier access of the transcription machinery for gene expression. This kind of modification is mediated by histone acetyltransferases, which add acetyl groups to activate gene expression, and histone deacetylases, which remove acetyl groups to inactivate gene expression. Kim et al. (2008) could show that AtWRKY38 and AtWRKY62, two negative regulators of plant defense, interact in the nucleus with histone deacetylase 19 (HDA19), a positive regulator of plant defense. Both WRKYs show transactivation activity in a reporter gene assay which is abolished by HDA19 suggesting that AtWRKY38 and AtWRKY62 induce the expression of genes negatively regulating plant defense and this is inhibited by HDA19. Epigenetic control was also observed for AtWRKY53 during senescence. For this WRKY gene, specific histone methylations are necessary for correct gene expression and progression of senescence (Ay et al., 2009). Methylation of histones can either activate or repress transcription depending on the methylated site mediated by histone methyltransferases and histone demethylases. Plants overexpressing SUVH2, a histone methyltransferase, have a different status of histone methylation, whereby the expression of AtWRKY53 is repressed (Ay et al., 2009). But also histone acetylation seems to be important for AtWRKY53 expression since the promoters of AtWRKY53 and AtWRKY6 are enriched with acetyl groups (Luna et al., 2012). Recently, yeast two-hybrid and bimolecular fluorescence complementation assays showed that banana MaWRKY1 could interact with MaHIS1, a linker histone H1 protein (Wang et al., 2012).

\section{WRKY FUNCTION CAN BE TRIGGERED BY SUBCELLULAR LOCALIZATION}

Most WRKY TFs are located in the nucleus for direct transcriptional regulation (Robatzek and Somssich, 2001; Zhang et al., 2004; Zheng et al., 2006; Liu et al., 2007b). However, an interesting example for WRKY TFs that regulate gene expression by changing their subcellular localization is given by Shang et al. (2010). Usually, WRKY40 inhibits expression of ABA-responsive genes in the nucleus. Triggered by high concentrations of ABA, AtWRKY40 interacts strongly with magnesium-protoporphyrin IX chelatase $\mathrm{H}$ subunit [CHLH]/putative ABA receptor (ABAR) inhibiting further regulatory function of AtWRKY40 in the nucleus. ABAR is localized predominantly in the outer chloroplast membrane, with its $\mathrm{N}$ - and C-terminus exposed to the cytosol. ABAR binds ABA and appears to be an ABA receptor (Shen et al., 2006). AtWRKY40 interaction with the C-terminus of ABAR in the cytosol releases inhibition of $\mathrm{ABA}$ response genes in the nucleus and $\mathrm{ABA}$ response can occur. Furthermore, the expression of AtWRKY40 is repressed after ABA treatment (Shang et al., 2010).

\section{CONCLUSION}

The hitherto characterized regulatory mechanisms controlling the function of TFs belonging to the bZIP and the WRKY families have been summarized in order to offer a comparative view. Not surprisingly, the major known mechanisms controlling protein activity have been found regulating members of both families. However, the prevalence of certain regulatory mechanisms reveals preferences in the manner how the activity of the proteins in each family is controlled, what we designate as a general regulatory strategy (Figure 5). In the case of the bZIPs, networking on the protein level by heterodimerization appears to be the preferred tool to adjust and fine-tune bZIP function. Regarding the WRKYs, controlling transcription of each other stands out as networking strategy for this family in synergism with the epigenetic control of their promoters.

It is tempting to speculate about the implications of using these different strategies. It can be argued that the bZIP strategy of heterodimerization with a strong component of post-translational regulation would enable very fast crosstalk between different input signals but at the same time imply keeping a pool of "ready to use" monomers. Such an energetically expensive strategy must grant counteracting advantages in order to be maintained during the evolution. Besides the fact that the bZIP strategy allows for 

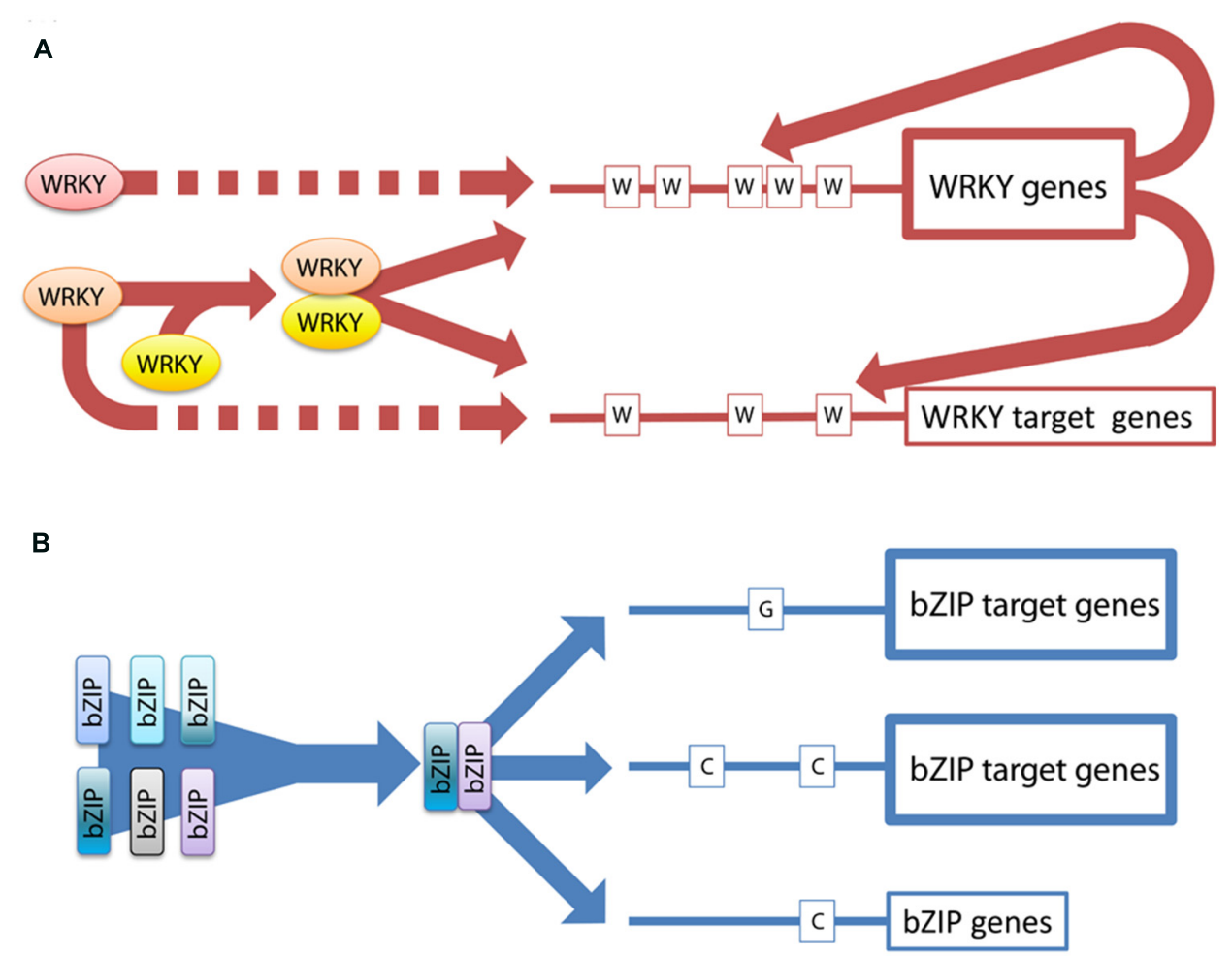

FIGURE 5 | Model featuring the main differences between the regulatory strategies of the two families. (A) The expression of WRKY TFs is strongly regulated at the transcriptional level. The promoters of the WRKY genes are enriched in W-boxes, which are bound by WRKY proteins indicating transcriptional networking and also feed-back regulations. The dotted lines indicate that WRKY possibly interact with the DNA also in a monomeric stage. In addition, WRKYs can form dimers and thereby increase the variability in regulating specific target genes. (B) In the bZIP family heterodimerization is extensively used to increase variability in target gene regulation. The activity of the bZIPs is regulated via specific dimerization which determines the target specificity. There are no indications for transcriptional networking. rapid responses, factor combination confers enhanced integration capacity and flexibility: a limited pool of monomers allows multitudes of responses. The looseness of the dimerization guarantees a certain degree of graduation and fine-tuning of several responses at the same time. In another sense, the WRKY strategy seems to actively strive for autocontrol by a decisive regulation of the own expression. Although this strategy results in slower responses, for it requires de novo synthesis of proteins, it ensures the proper timing and the steadiness of the response. These kinds of responses are expected to be rather long-term ones, so that they become buffered once they have been triggered. In agreement with these conjectures, bZIPs seem to have a more prominent role regarding stress adaptation, which require dynamic, adaptive responses; whereas WRKYs are frequently related to longer lasting situations, like pathogen defense or the senescence progression.

To sum up, we suggest that bZIPs and WRKYs follow different regulatory strategies and we hypothesize that these reveal different control methods, either the "adjustable kind" or the "slow-butsure" one. Although there are some facts which are undisputed, as the enrichment of WRKY binding sites in their own promoters, further data will be required to support our hypothesis. To this end, the identification and characterization of further response pathways involving WRKYs and bZIPs as well as system biology approaches combined with bioinformatics and modeling will help to unravel the network strategies of the two families in more depth. However, new in vivo approaches will be necessary to follow also the dynamic of these processes. In addition, deciphering molecular evolution of the two TF families in more detail might also provide inside into the strategies that these gene families pursue.

\section{ACKNOWLEDGMENTS}

We acknowledge financial support by the DFG, Ze 313/9-1 and the European Community, Framework 7, ITN MERIT

\section{REFERENCES}

Abe, M., Kobayashi, Y., Yamamoto, S., Daimon, Y., Yamaguchi, A., Ikeda, Y., et al. (2005). FD, a bZIP protein mediating signals from the floral pathway integrator FT at the shoot apex. Science 309, 1052-1056. doi: 10.1126/science.11 15983

Acharya, A., Ruvinov, S. B., Gal, J., Moll, J. R., and Vinson, C. (2002). A heterodimerizing leucine zipper coiled coil system for examining the specificity of a position interactions: amino acids I, V, L, N, A, and K. Biochemistry 41, 14122-14131. doi: 10.1021/bi020486r

Alonso, R., Onate-Sanchez, L., Weltmeier, F., Ehlert, A., Diaz, I., Dietrich, K., et al. (2009). A pivotal role of the basic leucine zipper transcription factor bZIP53 in the regulation of Arabidopsis seed maturation gene expression based on heterodimerization and protein complex formation. Plant Cell 21, 1747-1761. doi: 10.1105/tpc.108.062968

Alves, M., Dadalto, S., Gonçalves, A., De Souza, G., Barros, V., and Fietto, L. (2013). Plant bZIP transcription factors responsive to pathogens: a review. Int. J. Mol. Sci. 14, 7815-7828. doi: 10.3390/ijms14047815 
Amoutzias, G., Veron, A., Weiner, J., Robinson-Rechavi, M., Bornberg-Bauer, E., Oliver, S., et al. (2006). One billion years of bZIP transcription factor evolution: conservation and change in dimerization and DNA-binding site specificity. Mol. Biol. Evol. 24, 827-835. doi: 10.1093/molbev/msl211

Amoutzias, G. D., Robertson, D. L., Van De Peer, Y., and Oliver, S. G. (2008). Choose your partners: dimerization in eukaryotic transcription factors. Trends Biochem. Sci. 33, 220-229. doi: 10.1016/j.tibs.2008.02.002

Andronis, C., Barak, S., Knowles, S. M., Sugano, S., and Tobin, E. M. (2008) The clock protein CCA1 and the bZIP transcription factor HY5 physically interact to regulate gene expression in Arabidopsis. Mol. Plant 1, 58-67. doi: $10.1093 / \mathrm{mp} / \mathrm{ssm} 005$

Asai, T., Tena, G., Plotnikova, J., Willmann, M. R., Chiu, W. L., Gomez-Gomez, L., et al. (2002). MAP kinase signalling cascade in Arabidopsis innate immunity. Nature 415, 977-983. doi: 10.1038/415977a

Assunção, A. G. L., Herrero, E., Lin, Y.-F., Huettel, B., Talukdar, S., Smaczniak, C., et al. (2010). Arabidopsis thaliana transcription factors bZIP19 and bZIP23 regulate the adaptation to zinc deficiency. Proc. Natl. Acad. Sci. U.S.A. 107, 1029610301. doi: 10.1073/pnas.1004788107

Ay, N., Irmler, K., Fischer, A., Uhlemann, R., Reuter, G., and Humbeck, K. (2009). Epigenetic programming via histone methylation at WRKY53 controls leaf senescence in Arabidopsis thaliana. Plant J. 58, 333-346. doi: 10.1111/j.09607412.2009.03782.x

Babu, M. M., Iyer, L. M., Balaji, S., and Aravind, L. (2006). The natural history of the WRKY-GCM1 zinc fingers and the relationship between transcription factors and transposons. Nucleic Acids Res. 34, 6505-6520. doi: 10.1093/nar/gkl888

Babu Rajendra Prasad, V., Gupta, N., Nandi, A., and Chattopadhyay, S. (2012). HY1 genetically interacts with GBF1 and regulates the activity of the Z-box containing promoters in light signaling pathways in Arabidopsis thaliana. Mech. Dev. 129, 298-307. doi: 10.1016/j.mod.2012.06.004

Baena-González, E., Rolland, F., Thevelein, J. M., and Sheen, J. (2007). A central integrator of transcription networks in plant stress and energy signalling. Nature 448, 938-942. doi: 10.1038/nature06069

Bensmihen, S., Rippa, S., Lambert, G., Jublot, D., Pautot, V., Granier, F., et al. (2002). The homologous ABI5 and EEL transcription factors function antagonistically to fine-tune gene expression during late embryogenesis. Plant Cell 14, 1391-1403. doi: 10.1105/tpc.000869

Berri, S., Abbruscato, P., Faivre-Rampant, O., Brasileiro, A. C., Fumasoni, I., Satoh, K., et al. (2009). Characterization of WRKY co-regulatory networks in rice and Arabidopsis. BMC Plant Biol. 9:120. doi: 10.1186/1471-2229-9-120

Besseau, S., Li, J., and Palva, E. T. (2012). WRKY54 and WRKY70 co-operate as negative regulators of leaf senescence in Arabidopsis thaliana. J. Exp. Bot. 63, 2667-2679. doi: 10.1093/jxb/err450

Birkenbihl, R. P., Diezel, C., and Somssich, I. E. (2012). Arabidopsis WRKY33 is a key transcriptional regulator of hormonal and metabolic responses toward Botryti cinerea infection. Plant Physiol. 159, 266-285. doi: 10.1104/pp.111.192641

Brand, L. H., Fischer, N. M., Harter, K., Kohlbacher, O., and Wanke, D. (2013) Elucidating the evolutionary conserved DNA-binding specificities of WRKY transcription factors by molecular dynamics and in vitro binding assays. Nucleic Acids Res. 41, 9764-9778. doi: 10.1093/nar/gkt732

Breeze, E., Harrison, E., Mchattie, S., Hughes, L., Hickman, R., Hill, C., et al. (2011). High-resolution temporal profiling of transcripts during Arabidopsis leaf senescence reveals a distinct chronology of processes and regulation. Plant Cell 23, 873-894. doi: 10.1105/tpc.111.083345

Cai, M., Qiu, D., Yuan, T., Ding, X., Li, H., Duan, L., et al. (2008). Identification of novel pathogen-responsive cis-elements and their binding proteins in the promoter of OsWRKY13, a gene regulating rice disease resistance. Plant Cell Environ. 31, 86-96.

Chae, M. J., Lee, J. S., Nam, M. H., Cho, K., Hong, J. Y., Yi, S. A., et al. (2007). A rice dehydration-inducible SNF1-related protein kinase 2 phosphorylates an abscisic acid responsive element-binding factor and associates with ABA signaling. Plant Mol. Biol. 63, 151-169. doi: 10.1007/s11103-006-9079-x

Chen, H., Lai, Z., Shi, J., Xiao, Y., Chen, Z., and Xu, X. (2010). Roles of Arabidopsis WRKY18, WRKY40 and WRKY60 transcription factors in plant responses to abscisic acid and abiotic stress. BMC Plant Biol. 10:281. doi: 10.1186/1471-222910-281

Chen, Y.-T., Liu, H., Stone, S., and Callis, J. (2013). ABA and the ubiquitin E3 ligase KEEP ON GOING affect proteolysis of the Arabidopsis thaliana transcription factors ABF1 and ABF3. Plant J. 75, 965-976. doi: 10.1111/tpj.12259
Cheng, Y., Zhou, Y., Yang, Y., Chi, Y. J., Zhou, J., Chen, J. Y., et al. (2012). Structural and functional analysis of VQ motif-containing proteins in Arabidopsis as interacting proteins of WRKY transcription factors. Plant Physiol. 159, 810-825. doi: 10.1104/pp.112.196816

Chi, Y., Yang, Y., Zhou, Y., Zhou, J., Fan, B., Yu, J. Q., et al. (2013). Proteinprotein interactions in the regulation of WRKY transcription factors. Mol. Plant 6, 287-300. doi: 10.1093/mp/sst026

Cho, Y. H., Hong, J. W., Kim, E. C., and Yoo, S. D. (2012). Regulatory functions of SnRK1 in stress-responsive gene expression and in plant growth and development. Plant Physiol. 158, 1955-1964. doi: 10.1104/pp.111.189829

Choi, H. I., Hong, J., Ha, J., Kang, J., and Kim, S. Y. (2000). ABFs, a family of ABA-responsive element binding factors. J. Biol. Chem. 275, 1723-1730. doi: 10.1074/jbc.275.3.1723

Chujo, T., Miyamoto, K., Shimogawa, T., Shimizu, T., Otake, Y., Yokotani, N., et al. (2013). OsWRKY28, a PAMP-responsive transrepressor, negatively regulates innate immune responses in rice against rice blast fungus. Plant Mol. Biol. 82, 23-37. doi: 10.1007/s11103-013-0032-5

Ciolkowski, I., Wanke, D., Birkenbihl, R. P., and Somssich, I. E. (2008). Studies on DNA-binding selectivity of WRKY transcription factors lend structural clues into WRKY-domain function. Plant Mol. Biol. 68, 81-92. doi: 10.1007/s11103-0089353-1

Deppmann, C. D., Acharya, A., Rishi, V., Wobbes, B., Smeekens, S., Taparowsky, E. J., et al. (2004). Dimerization specificity of all 67 B-ZIP motifs in Arabidopsis thaliana: a comparison to Homo sapiens B-ZIP motifs. Nucleic Acids Res. 32, 3435-3445. doi: 10.1093/nar/gkh653

Deppmann, C. D., Alvania, R. S., and Taparowsky, E. J. (2006). Cross-species annotation of basic leucine zipper factor interactions: insight into the evolution of closed interaction networks. Mol. Biol. Evol. 23, 1480-1492. doi: 10.1093/molbev/msl022

Deppmann, C. D., Thornton, T. M., Utama, F. E., and Taparowsky, E. J. (2003). Phosphorylation of BATF regulates DNA binding: a novel mechanism for AP-1 (activator protein-1) regulation. Biochem. J. 374, 423-431. doi: 10.1042/BJ20030455

Dietrich, K., Weltmeier, F., Ehlert, A., Weiste, C., Stahl, M., Harter, K., et al. (2011). Heterodimers of the Arabidopsis transcription factors bZIP1 and bZIP53 reprogram amino acid metabolism during low energy stress. Plant Cell 23, 381-395. doi: $10.1105 /$ tpc. 110.075390

Djamei, A., Pitzschke, A., Nakagami, H., Rajh, I., and Hirt, H. (2007). Trojan horse strategy in Agrobacterium transformation: abusing MAPK defense signaling. Science 318, 453-456. doi: 10.1126/science.1148110

Dong, J., Chen, C., and Chen, Z. (2003). Expression profiles of the Arabidopsis WRKY gene superfamily during plant defense response. Plant Mol. Biol. 51, 21-37. doi: 10.1023/A:1020780022549

Duan, M. R., Nan, J., Liang, Y. H., Mao, P., Lu, L., Li, L., et al. (2007). DNA binding mechanism revealed by high resolution crystal structure of Arabidopsis thaliana WRKY1 protein. Nucleic Acids Res. 35, 1145-1154. doi: 10.1093/nar/ gkm001

Dyson, H. J., and Wright, P. E. (2005). Intrinsically unstructured proteins and their functions. Nat. Rev. Mol. Cell Biol. 6, 197-208. doi: 10.1038/nrm1589

Ehlert, A., Weltmeier, F., Wang, X., Mayer, C. S., Smeekens, S., VicenteCarbajosa, J., et al. (2006). Two-hybrid protein-protein interaction analysis in Arabidopsis protoplasts: establishment of a heterodimerization map of group C and group S bZIP transcription factors. Plant J. 46, 890-900. doi: 10.1111/j.1365-313X.2006.02731.x

Eulgem, T., Rushton, P. J., Robatzek, S., and Somssich, I. E. (2000). The WRKY superfamily of plant transcription factors. Trends Plant Sci. 5, 199-206. doi: 10.1016/S1360-1385(00)01600-9

Eulgem, T., Rushton, P. J., Schmelzer, E., Hahlbrock, K., and Somssich, I. E. (1999). Early nuclear events in plant defence signalling: rapid gene activation by WRKY transcription factors. EMBO J. 18, 4689-4699. doi: 10.1093/emboj/18. 17.4689

Fong, J., Keating, A., and Singh, M. (2004). Predicting specificity in bZIP coiled-coil protein interactions. Genome Biol. 5, R11. doi: 10.1186/gb-2004-5-2-r11

Foster, R., Izawa, T., and Chua, N.-H. (1994). Plant bZIP proteins gather at ACGT elements. FASEB J. 8, 192-200.

Fu, Z. Q., and Dong, X. (2013). Systemic acquired resistance: turning local infection into global defense. Annu. Rev. Plant Biol. 64, 839-863. doi: 10.1146/annurevarplant-042811-105606 
Fujii, Y., Shimizu, T., Toda, T., Yanagida, M., and Hakoshima, T. (2000). Structural basis for the diversity of DNA recognition by bZIP transcription factors. Nat. Struct. Biol. 7, 889-893. doi: 10.1038/82822

Fujita, Y., Fujita, M., Satoh, R., Maruyama, K., Parvez, M. M., Seki, M., et al. (2005). AREB1 is a transcription activator of novel ABRE-dependent ABA signaling that enhances drought stress tolerance in Arabidopsis. Plant Cell 17, 3470-3488. doi: 10.1105/tpc.105.035659

Gangappa, S. N., Crocco, C. D., Johansson, H., Datta, S., Hettiarachchi, C., Holm, M., et al. (2013). The Arabidopsis B-BOX protein BBX25 interacts with HY5, negatively regulating BBX22 expression to suppress seedling photomorphogenesis. Plant Cell 25, 1243-1257. doi: 10.1105/tpc.113.109751

Glawischnig, E. (2007). Camalexin. Phytochemistry 68, 401-406. doi: 10.1016/j.phytochem.2006.12.005

Glover, J. N. M., and Harrison, S. C. (1995). Crystal structure of the heterodimeric bZIP transcription factor c-Fos-c-Jun bound to DNA. Nature 373, 257-261. doi: $10.1038 / 373257 \mathrm{a} 0$

Hardtke, C. S., Gohda, K., Osterlund, M. T., Oyama, T., Okada, K., and Deng, X. W. (2000). HY5 stability and activity in Arabidopsis is regulated by phosphorylation in its COP1 binding domain. EMBO J. 19, 4997-5006. doi: 10.1093/emboj/19.18.4997

Holm, M., Ma, L.-G., Qu, L.-J., and Deng, X.-W. (2002). Two interacting bZIP proteins are direct targets of COP1-mediated control of light-dependent gene expression in Arabidopsis. Genes Dev. 16, 1247-1259. doi: 10.1101/gad.969702

Hsieh, T. H., Li, C. W., Su, R. C., Cheng, C. P., Sanjaya, Tsai, Y. C., et al. (2010). A tomato bZIP transcription factor, SlAREB, is involved in water deficit and salt stress response. Planta 231, 1459-1473. doi: 10.1007/s00425-010-1147-4

Hu, Y., Dong, Q., and Yu, D. (2012). Arabidopsis WRKY46 coordinates with WRKY70 and WRKY53 in basal resistance against pathogen Pseudomonas syringae. Plant Sci. 185-186, 288-297. doi: 10.1016/j.plantsci.2011.12.003

Huang, S., Gao, Y., Liu, J., Peng, X., Niu, X., Fei, Z., et al. (2012). Genome-wide analysis of WRKY transcription factors in Solanum lycopersicum. Mol. Genet. Genomics 287, 495-513. doi: 10.1007/s00438-012-0696-6

Hummel, M., Rahmani, F., Smeekens, S., and Hanson, J. (2009). Sucrose-mediated translational control. Ann. Bot. 104, 1-7. doi: 10.1093/aob/mcp086

Ishida, S., Yuasa, T., Nakata, M., and Takahashi, Y. (2008). A tobacco calciumdependent protein kinase, CDPK1, regulates the transcription factor REPRESSION OF SHOOT GROWTH in response to gibberellins. Plant Cell 20, 3273-3288. doi: 10.1105/tpc.107.057489

Ishiguro, S., and Nakamura, K. (1994). Characterization of a cDNA encoding a novel DNA-binding protein, SPF1, that recognizes SP8 sequences in the $5^{\prime}$ upstream regions of genes coding for sporamin and beta-amylase from sweet potato. Mol. Gen. Genet. 244, 563-571. doi: 10.1007/BF00282746

Ishihama, N., Yamada, R., Yoshioka, M., Katou, S., and Yoshioka, H. (2011). Phosphorylation of the Nicotiana benthamiana WRKY8 transcription factor by MAPK functions in the defense response. Plant Cell 23, 1153-1170. doi: 10.1105/tpc.110.081794

Iwata, Y., Fedoroff, N. V., and Koizumi, N. (2008). Arabidopsis bZIP60 is a proteolysis-activated transcription factor involved in the endoplasmic reticulum stress response. Plant Cell 20, 3107-3121. doi: 10.1105/tpc.108.061002

Izawa, T., Foster, R., and Chua, N.-H. (1993). Plant bZIP protein DNA binding specificity. J. Mol. Biol. 230, 1131-1144. doi: 10.1006/jmbi.1993.1230

Jakoby, M., Weisshaar, B., Dröge-Laser, W., Vicente-Carbajosa, J., Tiedemann, J., Kroj, T., et al. (2002). bZIP transcription factors in Arabidopsis. Trends Plant Sci. 7, 106-111. doi: 10.1016/S1360-1385(01)02223-3

Jiang, Y., and Deyholos, M. K. (2009). Functional characterization of Arabidopsis $\mathrm{NaCl}$-inducible WRKY25 and WRKY33 transcription factors in abiotic stresses. Plant Mol. Biol. 69, 91-105. doi: 10.1007/s11103-008-9408-3

Johnson, C. S., Kolevski, B., and Smyth, D. R. (2002). TRANSPARENT TESTA GLABRA2, a trichome and seed coat development gene of Arabidopsis, encodes a WRKY transcription factor. Plant Cell 14, 1359-1375. doi: 10.1105/tpc. 001404

Journot-Catalino, N., Somssich, I. E., Roby, D., and Kroj, T. (2006). The transcription factors WRKY11 and WRKY17 act as negative regulators of basal resistance in Arabidopsis thaliana. Plant Cell 18, 3289-3302. doi: 10.1105/tpc.106.044149

Kagaya, Y., Hobo, T., Murata, M., Ban, A., and Hattori, T. (2002). Abscisic acid-induced transcription is mediated by phosphorylation of an abscisic acid response element binding factor, TRAB1. Plant Cell 14, 3177-3189. doi: $10.1105 /$ tpc.005272
Kaminaka, H., Nake, C., Epple, P., Dittgen, J., Schutze, K., Chaban, C., et al. (2006) bZIP10-LSD1 antagonism modulates basal defense and cell death in Arabidopsis following infection. EMBO J. 25, 4400-4411. doi: 10.1038/sj.emboj.7601312

Kang, S. G., Price, J., Lin, P. C., Hong, J. C., and Jang, J. C. (2010). The Arabidopsis bZIP1 transcription factor is involved in sugar signaling, protein networking, and DNA binding. Mol. Plant 3, 361-373. doi: 10.1093/mp/ssp115

Kim, K. C., Lai, Z., Fan, B., and Chen, Z. (2008). Arabidopsis WRKY38 and WRKY62 transcription factors interact with histone deacetylase 19 in basal defense. Plant Cell 20, 2357-2371. doi: 10.1105/tpc.107.055566

Kirchler, T., Briesemeister, S., Singer, M., Schütze, K., Keinath, M., Kohlbacher, O., et al. (2010). The role of phosphorylatable serine residues in the DNA-binding domain of Arabidopsis bZIP transcription factors. Eur. J. Cell Biol. 89, 175-183. doi: 10.1016/j.ejcb.2009.11.023

Kobayashi, Y., Murata, M., Minami, H., Yamamoto, S., Kagaya, Y., Hobo, T., et al. (2005). Abscisic acid-activated SNRK2 protein kinases function in the gene-regulation pathway of $\mathrm{ABA}$ signal transduction by phosphorylating $\mathrm{ABA}$ response element-binding factors. Plant J. 44, 939-949. doi: 10.1111/j.1365313X.2005.02583.x

Konig, P., and Richmond, T. J. (1993). The X-ray structure of the GCN4-bZIP bound to ATF/CREB site DNA shows the complex depends on DNA flexibility. J. Mol. Biol. 233, 139-154. doi: 10.1006/jmbi.1993.1490

Krylov, D., Mikhailenko, I., and Vinson, C. (1994). A thermodynamic scale for leucine zipper stability and dimerization specificity: e and $\mathrm{g}$ interhelical interactions. EMBO J. 13, 2849-2861.

Kuo, M.-H., Vom Baur, E., Struhl, K., and Allis, C. D. (2000). Gen4 activator targets Gen5 histone acetyltransferase to specific promoters independently of transcription. Mol. Cell. 6, 1309-1320. doi: 10.1016/S1097-2765(00) 00129-5

Lai, Z., Li, Y., Wang, F., Cheng, Y., Fan, B., Yu, J. Q., et al. (2011). Arabidopsis sigma factor binding proteins are activators of the WRKY33 transcription factor in plant defense. Plant Cell 23, 3824-3841. doi: 10.1105/tpc.111.090571

Lee, S., Shuman, J. D., Guszczynski, T., Sakchaisri, K., Sebastian, T., Copeland, T. D., et al. (2010). RSK-mediated phosphorylation in the C/EBP leucine zipper regulates DNA binding, dimerization, and growth arrest activity. Mol. Cell. Biol. 30, 2621-2635. doi: 10.1128/MCB.00782-09

Li, W., Jain, M. R., Chen, C., Yue, X., Hebbar, V., Zhou, R., et al. (2005). Nrf2 possesses a redox-insensitive nuclear export signal overlapping with the leucine zipper motif. J. Biol. Chem. 280, 28430-28438. doi: 10.1074/jbc.M410601200

Liu, J. X., Srivastava, R., Che, P., and Howell, S. H. (2007a). An endoplasmic reticulum stress response in Arabidopsis is mediated by proteolytic processing and nuclear relocation of a membrane-associated transcription factor, bZIP28. Plant Cell 19, 4111-4119. doi: 10.1105/tpc.106.050021

Liu, X., Bai, X., Wang, X., and Chu, C. (2007b). OsWRKY71, a rice transcription factor, is involved in rice defense response. J. Plant Physiol. 164, 969-979. doi: 10.1016/j.jplph.2006.07.006

Liu, J.-X., Srivastava, R., and Howell, S. H. (2008). Stress-induced expression of an activated form of AtbZIP17 provides protection from salt stress in Arabidopsis. Plant Cell Environ. 31, 1735-1743. doi: 10.1111/j.1365-3040.2008.01873.x

Liu, Z. Q., Yan, L., Wu, Z., Mei, C., Lu, K., Yu, Y. T., et al. (2012). Cooperation of three WRKY-domain transcription factors WRKY18, WRKY40, and WRKY60 in repressing two ABA-responsive genes ABI4 and ABI5 in Arabidopsis. J. Exp. Bot. 63, 6371-6392. doi: 10.1093/jxb/ers293

Luna, E., Bruce, T. J., Roberts, M. R., Flors, V., and Ton, J. (2012). Nextgeneration systemic acquired resistance. Plant Physiol. 158, 844-853. doi: 10.1104/pp.111.187468

Maeo, K., Hayashi, S., Kojima-Suzuki, H., Morikami, A., and Nakamura, K. (2001). Role of conserved residues of the WRKY domain in the DNA-binding of tobacco WRKY family proteins. Biosci. Biotechnol. Biochem. 65, 2428-2436. doi: 10.1271/bbb.65.2428

Mallappa, C., Singh, A., Ram, H., and Chattopadhyay, S. (2008). GBF1, a transcription factor of blue light signaling in Arabidopsis, is degraded in the dark by a proteasome-mediated pathway independent of COP1 and SPA1. J. Biol. Chem. 283, 35772-35782. doi: 10.1074/jbc.M803437200

Mangelsen, E., Kilian, J., Berendzen, K. W., Kolukisaoglu, U. H., Harter, K., Jansson, C., et al. (2008). Phylogenetic and comparative gene expression analysis of barley (Hordeum vulgare) WRKY transcription factor family reveals putatively retained functions between monocots and dicots. BMC Genomics 9:194. doi: 10.1186/1471-2164-9-194 
Mao, G., Meng, X., Liu, Y., Zheng, Z., Chen, Z., and Zhang, S. (2011). Phosphorylation of a WRKY transcription factor by two pathogen-responsive MAPKs drives phytoalexin biosynthesis in Arabidopsis. Plant Cell 23, 1639-1653. doi: 10.1105/tpc.111.084996

Matiolli, C. C., Tomaz, J. P., Duarte, G. T., Prado, F. M., Del Bem, L. E. V., Silveira, A. B., et al. (2011). The Arabidopsis bZIP gene AtbZIP63 is a sensitive integrator of transient abscisic acid and glucose signals. Plant Physiol. 157, 692-705. doi: $10.1104 /$ pp.111.181743

Menkens, A. E., and Cashmore, A. R. (1994). Isolation and characterization of a fourth Arabidopsis thaliana G-box-binding factor, which has similarities to Fos oncoprotein. Proc. Natl. Acad. Sci. U.S.A. 91, 2522-2526. doi: 10.1073/pnas.91.7.2522

Miao, Y., Laun, T., Zimmermann, P., and Zentgraf, U. (2004). Targets of the WRKY53 transcription factor and its role during leaf senescence in Arabidopsis. Plant Mol. Biol. 55, 853-867. doi: 10.1007/s11103-005-2142-1

Miao, Y., Laun, T. M., Smykowski, A., and Zentgraf, U. (2007). Arabidopsis MEKK1 can take a short cut: it can directly interact with senescence-related WRKY53 transcription factor on the protein level and can bind to its promoter. Plant Mol. Biol. 65, 63-76. doi: 10.1007/s11103-007-9198-z

Miller, M. (2009). The importance of being flexible: the case of basic region leucine zipper transcriptional regulators. Curr. Protein Pept. Sci. 10, 244-269. doi: 10.2174/138920309788452164

Miller, M., Shuman, J. D., Sebastian, T., Dauter, Z., and Johnson, P. F. (2003). Structural basis for DNA recognition by the basic region leucine zipper transcription factor CCAAT/enhancer-binding protein alpha. J. Biol. Chem. 278, 15178-15184. doi: 10.1074/jbc.M300417200

Miotto, B., and Struhl, K. (2006). Differential gene regulation by selective association of transcriptional coactivators and bZIP DNA-binding domains. Mol. Cell. Biol. 26, 5969-5982. doi: 10.1128/MCB.00696-06

Moitra, J., Szilák, L., Krylov, D., and Vinson, C. (1997). Leucine is the most stabilizing aliphatic amino acid in the $\mathrm{d}$ position of a dimeric leucine zipper coiled coil. Biochemistry 36, 12567-12573. doi: 10.1021/bi971424h

Newman, J. R. S., and Keating, K. (2003). Comprehensive identification of human bZIP interactions with coiled-coil arrays. Science 300, 2097-2101. doi: 10.1126/science. 1084648

Nieva, C., Busk, P. K., Domínguez-Puigjaner, E., Lumbreras, V., Testillano, P. S., Risueño, M.-C., et al. (2005). Isolation and functional characterisation of two new bZIP maize regulators of the ABA responsive gene rab28. Plant Mol. Biol. 58, 899-914. doi: 10.1007/s11103-005-8407-x

Nijhawan, A., Jain, M., Tyagi, A. K., and Khurana, J. P. (2007). Genomic survey and gene expression analysis of the basic leucine zipper transcription factor family in rice. Plant Physiol. 146, 333-350. doi: 10.1104/pp.107.112821

Niu, X., Renshaw-Gegg, L., Miller, L., and Guiltinan, M. J. (1999). Bipartite determinants of DNA-binding specificity of plant basic leucine zipper proteins. Plant Mol. Biol. 41, 1-13. doi: 10.1023/A:1006206011502

Oliphant, A. R., Brandl, C. J., and Struhl, K. (1989). Defining the sequence specificity of DNA-binding proteins by selecting binding sites from random-sequence oligonucleotides: analysis of yeast GCN4 protein. Mol. Cell. Biol. 9, 2944-2949.

Orellana, S., Yanez, M., Espinoza, A., Verdugo, I., Gonzalez, E., Ruiz-Lara, S., et al. (2010). The transcription factor SIAREB1 confers drought, salt stress tolerance and regulates biotic and abiotic stress-related genes in tomato. Plant Cell Environ. 33, 2191-2208. doi: 10.1111/j.1365-3040.2010.02220.x

Pandey, S. P., and Somssich, I. E. (2009). The role of WRKY transcription factors in plant immunity. Plant Physiol. 150, 1648-1655. doi: 10.1104/pp.109.138990

Petitot, A. S., Barsalobres-Cavallari, C., Ramiro, D., Albuquerque Freire, E., Etienne, H., and Fernandez, D. (2013). Promoter analysis of the WRKY transcription factors CaWRKYla and CaWRKY1b homoeologous genes in coffee (Coffea arabica). Plant Cell Rep. 32, 1263-1276. doi: 10.1007/s00299-013-1440-3

Pontier, D., Privat, I., Trifa, Y., Zhou, J.-M., Klessig, D. F., and Lam, E. (2002). Differential regulation of TGA transcription factors by post-transcriptional control. Plant J. 32, 641-653. doi: 10.1046/j.1365-313X.2002.01461.x

Popescu, S. C., Popescu, G. V., Bachan, S., Zhang, Z., Gerstein, M., Snyder, M., et al. (2009). MAPK target networks in Arabidopsis thaliana revealed using functional protein microarrays. Genes Dev. 23, 80-92. doi: 10.1101/gad.1740009

Potschin, M., Schlienger, S., Bieker, S., and Zentgraf, U. (2013). Senescence networking: WRKY18 is an upstream regulator, a downstream target gene, and a protein interaction partner of WRKY53. J. Plant Growth Regul. 33, 106-118. doi: $10.1007 / \mathrm{s} 00344-013-9380-2$
Ren, D., Liu, Y., Yang, K. Y., Han, L., Mao, G., Glazebrook, J., et al. (2008). A fungalresponsive MAPK cascade regulates phytoalexin biosynthesis in Arabidopsis. Proc. Natl. Acad. Sci. U.S.A. 105, 5638-5643. doi: 10.1073/pnas.0711301105

Robatzek, S., and Somssich, I. E. (2001). A new member of the Arabidopsis WRKY transcription factor family, AtWRKY6, is associated with both senescenceand defence-related processes. Plant J. 28, 123-133. doi: 10.1046/j.1365313X.2001.01131.x

Robatzek, S., and Somssich, I. E. (2002). Targets of AtWRKY6 regulation during plant senescence and pathogen defense. Genes Dev. 16, 1139-1149. doi: 10.1101/gad.222702

Rochon, A., Boyle, P., Wignes, T., Fobert, P. R., and Despres, C. (2006). The coactivator function of Arabidopsis NPR1 requires the core of its BTB/POZ domain and the oxidation of C-terminal cysteines. Plant Cell 18, 3670-3685. doi: $10.1105 /$ tpc. 106.046953

Rook, F., Weisbeek, P., and Smeekens, S. (1998). The light-regulated Arabidopsis bZIP transcription factor gene ATB2 encodes a protein with an unusually long leucine zipper domain. Plant Mol. Biol. 37, 171-178. doi: 10.1023/A:1005964327725

Rushton, D. L., Tripathi, P., Rabara, R. C., Lin, J., Ringler, P., Boken, A. K., et al. (2012). WRKY transcription factors: key components in abscisic acid signalling. Plant Biotechnol. J. 10, 2-11. doi: 10.1111/j.1467-7652.2011.00634.x

Rushton, P. J., Macdonald, H., Huttly, A. K., Lazarus, C. M., and Hooley, R. (1995). Members of a new family of DNA-binding proteins bind to a conserved ciselement in the promoters of alpha-Amy2 genes. Plant Mol. Biol. 29, 691-702. doi: 10.1007/BF00041160

Rushton, P. J., Somssich, I. E., Ringler, P., and Shen, Q. J. (2010). WRKY transcription factors. Trends Plant Sci. 15, 247-258. doi: 10.1016/j.tplants.2010.02.006

Rushton, P. J., Torres, J. T., Parniske, M., Wernert, P., Hahlbrock, K., and Somssich, I. E. (1996). Interaction of elicitor-induced DNA-binding proteins with elicitor response elements in the promoters of parsley PR1 genes. EMBO J. 15, 5690-5700.

Scarpeci, T. E., Zanor, M. I., Mueller-Roeber, B., and Valle, E. M. (2013). Overexpression of AtWRKY30 enhances abiotic stress tolerance during early growth stages in Arabidopsis thaliana. Plant Mol. Biol. 83, 265-277. doi: 10.1007/s11103-013-0090-8

Schumacher, M. A., Goodman, R. H., and Brennan, R. G. (2000). The structure of a CREB bZIPmiddle dotSomatostatin CRE complex reveals the basis for selective dimerization and divalent cation-enhanced DNA binding. J. Biol. Chem. 275, 35242-35247. doi: 10.1074/jbc.M007293200

Seldeen, K. L., Mcdonald, C. B., Deegan, B. J., and Farooq, A. (2008). Coupling of folding and DNA-binding in the bZIP domains of Jun-Fos heterodimeric transcription factor. Arch. Biochem. Biophys. 473, 48-60. doi: 10.1016/j.abb.2008.02.024

Shaikhali, J., Noren, L., de Dios Barajas-Lopez, J., Srivastava, V., Konig, J., Sauer, U. H., et al. (2012). Redox-mediated mechanisms regulate DNA binding activity of the G-group of basic region leucine zipper (bZIP) transcription factors in Arabidopsis. J. Biol. Chem. 287, 27510-27525. doi: 10.1074/jbc.M112. 361394

Shang, Y., Yan, L., Liu, Z. Q., Cao, Z., Mei, C., Xin, Q., et al. (2010). The Mg-chelatase $\mathrm{H}$ subunit of Arabidopsis antagonizes a group of WRKY transcription repressors to relieve ABA-responsive genes of inhibition. Plant Cell 22, 1909-1935. doi: 10.1105/tpc. 110.073874

Shen, H., Cao, K., and Wang, X. (2007). A conserved proline residue in the leucine zipper region of AtbZIP34 and AtbZIP61 in Arabidopsis thaliana interferes with the formation of homodimer. Biochem. Biophys. Res. Commun. 362, 425-430. doi: 10.1016/j.bbrc.2007.08.026

Shen, H., Cao, K., and Wang, X. (2008). AtbZIP16 and AtbZIP68, two new members of GBFs, can interact with other G group bZIPs in Arabidopsis thaliana. BMB Rep. 41, 132-138. doi: 10.5483/BMBRep.2008.41.2.132

Shen, Y. Y., Wang, X. F., Wu, F. Q., Du, S. Y., Cao, Z., Shang, Y., et al. (2006). The $\mathrm{Mg}$-chelatase $\mathrm{H}$ subunit is an abscisic acid receptor. Nature 443, 823-826. doi: 10.1038/nature05176

Singh, A., Ram, H., Abbas, N., and Chattopadhyay, S. (2012). Molecular interactions of GBF1 with HY5 and HYH proteins during light-mediated seedling development in Arabidopsis thaliana. J. Biol. Chem. 287, 25995-26009. doi: 10.1074/jbc.M111.333906

Sirichandra, C., Davanture, M., Turk, B. E., Zivy, M., Valot, B., Leung, J., et al. (2010). The Arabidopsis ABA-activated kinase OST1 phosphorylates the bZIP transcription factor $\mathrm{ABF} 3$ and creates a 14-3-3 binding site involved in its turnover. PLoS ONE 5:e13935. doi: 10.1371/journal.pone.0013935 
Smykowski, A., Zimmermann, P., and Zentgraf, U. (2010). G-box binding factor 1 reduces CATALASE2 expression and regulates the onset of leaf senescence in Arabidopsis. Plant Physiol. 153, 1321-1331. doi: 10.1104/pp.110.157180

Suttipanta, N., Pattanaik, S., Kulshrestha, M., Patra, B., Singh, S. K., and Yuan, L. (2011). The transcription factor CrWRKY1 positively regulates the terpenoid indole alkaloid biosynthesis in Catharanthus roseus. Plant Physiol. 157, 20812093. doi: 10.1104/pp.111.181834

Tsugama, D., Liu, S., and Takano, T. (2012). A bZIP protein, VIP1, is a regulator of osmosensory signaling in Arabidopsis. Plant Physiol. 159, 144-155. doi: $10.1104 /$ pp.112.197020

Turck, F., Zhou, A., and Somssich, I. E. (2004). Stimulus-dependent, promoterspecific binding of transcription factor WRKY1 to Its native promoter and the defense-related gene PcPR1-1 in Parsley. Plant Cell 16, 2573-2585. doi: 10.1105/tpc.104.024810

Ulker, B., and Somssich, I. E. (2004). WRKY transcription factors: from DNA binding towards biological function. Curr. Opin. Plant Biol. 7, 491-498. doi: 10.1016/j.pbi.2004.07.012

Uno, Y., Furihata, T., Abe, H., Yoshida, R., Shinozaki, K., and Yamaguchi-Shinozaki, K. (2000). Arabidopsis basic leucine zipper transcription factors involved in an abscisic acid-dependent signal transduction pathway under drought and high-salinity conditions. Proc. Natl. Acad. Sci. U.S.A. 97, 11632-11637. doi: 10.1073/pnas.190309197

Vinson, C., Acharya, A., and Taparowsky, E. J. (2006). Deciphering B-ZIP transcription factor interactions in vitro and in vivo. Biochim. Biophys. Acta 1759, 4-12. doi: 10.1016/j.bbaexp.2005.12.005

Vinson, C., Myakishev, M., Acharya, A., Mir, A. A., Moll, J. R., and Bonovich, M. (2002). Classification of human B-ZIP proteins based on dimerization properties. Mol. Cell. Biol. 22, 6321-6335. doi: 10.1128/MCB.22.18.6321-6335.2002

Wang, C., Deng, P., Chen, L., Wang, X., Ma, H., Hu, W., et al. (2013). A wheat WRKY transcription factor TaWRKY10 confers tolerance to multiple abiotic stresses in transgenic tobacco. PLoS ONE 8:e65120. doi: 10.1371/journal.pone. 0065120

Wang, D., Amornsiripanitch, N., and Dong, X. (2006). A genomic approach to identify regulatory nodes in the transcriptional network of systemic acquired resistance in plants. PLoS Pathog. 2:e123. doi: 10.1371/journal.ppat.00 20123

Wang, J. N., Kuang, J. F., Shan, W., Chen, J., Xie, H., Lu, W. J., et al. (2012) Expression profiles of a banana fruit linker histone $\mathrm{H} 1$ gene MaHIS1 and its interaction with a WRKY transcription factor. Plant Cell Rep. 31, 1485-1494. doi: 10.1007/s00299-012-1263-7

Wei, K., Chen, J., Wang, Y., Chen, Y., Chen, S., Lin, Y., et al. (2012). Genomewide analysis of bZIP-encoding genes in maize. DNA Res. 19, 463-476. doi: 10.1093/dnares/dss026

Weltmeier, F., Ehlert, A., Mayer, C. S., Dietrich, K., Wang, X., Schutze, K., et al. (2006). Combinatorial control of Arabidopsis proline dehydrogenase transcription by specific heterodimerisation of bZIP transcription factors. EMBO J. 25 3133-3143. doi: 10.1038/sj.emboj.7601206

Weltmeier, F., Rahmani, F., Ehlert, A., Dietrich, K., Schütze, K., Wang, X., et al (2008). Expression patterns within the Arabidopsis C/S1 bZIP transcription factor network: availability of heterodimerization partners controls gene expression during stress response and development. Plant Mol. Biol. 69, 107-119. doi: 10.1007/s11103-008-9410-9

Wiese, A., Elzinga, N., Wobbes, B., and Smeekens, S. (2004). A conserved upstream open reading frame mediates sucrose-induced repression of translation. Plant Cell 16, 1717-1729. doi: 10.1105/tpc.019349
Williams, M. E., Foster, R., and Chua, N.-H. (1992). Sequences flanking the hexameric G-box core CACGTG affect the specificity of protein binding. Plant Cell 4, 485-496. doi: 10.1105/tpc.4.4.485

Xu, X., Chen, C., Fan, B., and Chen, Z. (2006). Physical and functional interactions between pathogen-induced Arabidopsis WRKY18, WRKY40, and WRKY60 transcription factors. Plant Cell 18, 1310-1326. doi: 10.1105/tpc.105.037523

Yamada, Y., and Sato, F. (2013). Transcription factors in alkaloid biosynthesis. Int. Rev. Cell Mol. Biol. 305, 339-382. doi: 10.1016/B978-0-12-407695-2.00008-1

Yamasaki, K., Kigawa, T., Inoue, M., Tateno, M., Yamasaki, T., Yabuki, T., et al. (2005). Solution structure of an Arabidopsis WRKY DNA binding domain. Plant Cell 17, 944-956. doi: 10.1105/tpc.104.026435

Yamasaki, K., Kigawa, T., Watanabe, S., Inoue, M., Yamasaki, T., Seki, M., et al. (2012). Structural basis for sequence-specific DNA recognition by an Arabidopsis WRKY transcription factor. J. Biol. Chem. 287, 7683-7691. doi: 10.1074/jbc.M111.279844

Yang, Z., Patra, B., Li, R., Pattanaik, S., and Yuan, L. (2013). Promoter analysis reveals cis-regulatory motifs associated with the expression of the WRKY transcription factor CrWRKY1 in Catharanthus roseus. Planta. doi: 10.1007/s00425-013-1949-2 [Epub ahead of print].

Zentgraf, U., Laun, T., and Miao, Y. (2010). The complex regulation of WRKY53 during leaf senescence of Arabidopsis thaliana. Eur. J. Cell Biol. 89, 133-137. doi: 10.1016/j.ejcb.2009.10.014

Zhang, Y., and Wang, L. (2005). The WRKY transcription factor superfamily: its origin in eukaryotes and expansion in plants. BMC Evol. Biol. 5:1. doi: $10.1186 / 1471-2148-5-1$

Zhang, Z. L., Xie, Z., Zou, X., Casaretto, J., Ho, T. H., and Shen, Q. J. (2004). A rice WRKY gene encodes a transcriptional repressor of the gibberellin signaling pathway in aleurone cells. Plant Physiol. 134, 1500-1513. doi: 10.1104/pp.103.034967

Zheng, Z., Qamar, S. A., Chen, Z., and Mengiste, T. (2006). Arabidopsis WRKY33 transcription factor is required for resistance to necrotrophic fungal pathogens. Plant J. 48, 592-605. doi: 10.1111/j.1365-313X.2006.02901.x

Zhou, X., Jiang, Y., and Yu, D. (2011). WRKY22 transcription factor mediates dark-induced leaf senescence in Arabidopsis. Mol. Cells 31, 303-313. doi: 10.1007/s10059-011-0047-1

Zou, M., Guan, Y., Ren, H., Zhang, F., and Chen, F. (2007). Characterization of alternative splicing products of bZIP transcription factors OsABI5. Biochem. Biophys. Res. Commun. 360, 307-313. doi: 10.1016/j.bbrc.2007.05.226

Conflict of Interest Statement: The authors declare that the research was conducted in the absence of any commercial or financial relationships that could be construed as a potential conflict of interest.

Received: 17 December 2013; accepted: 08 April 2014; published online: 30 April 2014 Citation: Llorca CM, Potschin M and Zentgraf U (2014) bZIPs and WRKYs: two large transcription factor families executing two different functional strategies. Front. Plant Sci. 5:169. doi: 10.3389/fpls.2014.00169

This article was submitted to Plant Systems Biology, a section of the journal Frontiers in Plant Science.

Copyright (C) 2014 Llorca, Potschin and Zentgraf. This is an open-access article distributed under the terms of the Creative Commons Attribution License (CC BY). The use, distribution or reproduction in other forums is permitted, provided the original author(s) or licensor are credited and that the original publication in this journal is cited, in accordance with accepted academic practice. No use, distribution or reproduction is permitted which does not comply with these terms. 